\title{
The Boost Problem in General Relativity
}

\author{
D. Christodoulou ${ }^{1}$ and N. O'Murchadha ${ }^{2}$ \\ 1 Max-Planck-Institut für Physik und Astrophysik, Institut für Astrophysik, D-8046 Garching bei \\ München, Federal Republic Germany \\ 2 Physics Department, University College, Cork, Ireland
}

\begin{abstract}
We show that any asymptotically flat initial data for the Einstein field equations have a development which includes complete spacelike surfaces boosted relative to the initial surface. Furthermore, the asymptotic fall off is preserved along these boosted surfaces and there exists a global system of harmonic coordinates on such a development. We also extend former results on global solutions of the constraint equations. By virtue of this extension, the constraint and evolution parts of the problem fit together exactly. Several theorems are given which concern the behaviour in the large of general classes of linear and quasilinear differential systems. This paper contains in addition a systematic exposition of the functional spaces employed.
\end{abstract}

\section{Introduction}

Lichnerowicz showed [1] that the Einstein equations form a system in involution and that the Cauchy problem in general relativity consists of two problems: the problem of the initial value constraints and the problem of evolution. ChoquetBruhat [2] employed the harmonic coordinate condition to reduce the evolution equations to a hyperbolic system and showed the existence and uniqueness of local-in-time solutions. An improvement of this result was later obtained in [3] based on the work of Leray [4] and Dionne [5] on hyperbolic systems. Global uniqueness, namely existence of a unique maximal development of the initial data, was shown by Choquet-Bruhat and Geroch in [6]. The evolution equations were studied from the point of view of a first order symmetric hyperbolic system by Fischer and Marsden [7]. The sharpest differentiability results for the evolution equations are due to Hughes, Kato and Marsden [8]. The problem of the initial value constraints, formulated in a particularly satisfactory way in the work of York [9] and O'Murchadha and York [10], was studied on a compact manifold by Choquet-Bruhat [11], and on a manifold which is euclidean at infinity by Cantor $[12,13]$ and Chaljub-Simon and Choquet-Bruhat [14].

The existence of local-in-time solutions to the Einstein equations is a result which is perhaps sufficient for the study of cosmology. It is however insufficient for 
the study of isolated systems. With this in mind, we wish to pose a sequence of three problems for the vacuum Einstein equations on $\mathbb{R}^{4}$ which are extensions of the local-in-time problem in a successively stronger sense. In each case, the first part of the problem is to construct on the surface $x^{0}=0$, which we call $\Sigma$, a complete riemannian metric $g$ and a 2-covariant symmetric tensorfield $k$, such that the pair $(g, k)$ satisfy the initial value constraints. In each case also, we are asked to find an open set $\Omega \supset \Sigma$ and a solution $\gamma$ to the vacuum Einstein equations on $\Omega$ such that $(g, k)$ are respectively the first and second fundamental forms of $\Sigma$ relative to $\gamma$ and $(\Omega, \gamma)$ is a globally hyperbolic spacetime. This is what is meant by a development of the initial data $(\Sigma, g, k)$. The sequence of three problems which we shall pose are characterized by successively stronger demands on the largeness of $\Omega$. To be precise, let us consider a pair of points $p, q \in \Omega$ and let us define $\chi^{+}(p, q)$ to be the supremum of the lengths of all future-directed non-spacelike curves from $p$ to $q$ if $q \in J^{+}(p)$ (the causal future of $p$ ) and zero otherwise. Thus $\chi^{+}(p, q)$ is the future temporal distance of $q$ from $p$. Similarly, we may define the past temporal distance $\chi^{-}(p, q)$. If $(\Omega, \gamma)$ is globally hyperbolic, $\chi^{+}$and $\chi^{-}$are known [15] to be continuous maps $\Omega \times \Omega \rightarrow \mathbb{R}^{+}$. Let us also define the function $\chi^{+}$on $\Omega$, taking values on the extended positive real line by:

$$
\tilde{\chi}^{+}(p)=\sup _{q \in \Omega} \chi^{+}(p, q)
$$

Thus $\tilde{\chi}^{+}(p)$ is the future temporal extent of $\Omega$ at $p$, namely the future temporal distance of the boundary of $\Omega$ from $p$. We may define the function $\tilde{\chi}^{-}$(past temporal extent) in a similar way. Let $d(x, y)$ be the distance function on $(\Sigma, g)$ defined by its riemannian structure and let $\tilde{d}(x)=d(x, 0)$ where 0 is a fixed point on $\Sigma$ which we take as the origin. We denote by $\bar{B}_{R}=\{x \in \Sigma \mid \tilde{d}(x) \leqq R\}$ the closed ball of radius $R$ centered at 0 on $(\Sigma, g)$.

1. The boost problem: $(\Omega, \gamma)$ should be such that there exists a $\lambda>0$ such that $\forall x \in \Sigma:$

$$
\tilde{\chi}^{ \pm}(x)>\lambda \tilde{d}(x)
$$

Thus we require the temporal extent of $(\Omega, \gamma)$ to grow linearly with the radial distance. We have given to this problem the name "boost problem" because in this case $(\Omega, \gamma)$ includes complete Cauchy surfaces wich are "boosted" relative to $\Sigma$.

2. The radiation problem: $(\Omega, \gamma)$ should be such that there exists a (finite) real number $R$ such that $\forall x \in \Sigma-\bar{B}_{R}$

$$
\tilde{\chi}^{ \pm}(x)=\infty
$$

In this case there are complete null geodesics in $(\Omega, \gamma)$ through every point of $\Sigma-\bar{B}_{R}$, a fact which justifies the name "radiation problem." This problem is of great interest for the study of isolated systems since it should allow one to investigate the asymptotic behaviour of the solution along the light rays. Finally, we can pose:

3. The global problem: $(\Omega, \gamma)$ should be timelike and null geodesically complete. From the singularity theorems [15], we know well that this problem can have solutions only for limited initial data. 
In this paper we shall give a complete solution to the boost problem for freely specifiable initial data which are asymptotically flat in a very weak sense. In retrospect this result is not surprising because singularities are expected to form only in regions of strong gravitational fields. Since the data falls off at infinity, one should be able to go out far enough to find a region of sufficiently weak data where the time interval of existence is long. However it is not at all clear, a priori, that this time interval grows linearly with the radial distance. In fact it will be seen that the Yang-Mills equations have very different asymptotic behaviour. Our results are significant for the study of spacelike infinity [16-19].

One of the strengths of the methods used in this paper is that the function spaces in which solutions to the initial value constraints are constructed are exactly those used to construct solutions to the evolution equations. This precise fitting of the elliptic and hyperbolic parts of the problem had been achieved only for the completely local Cauchy problem, namely local-in-time and local-in-space.

We have tried to give a self-contained exposition.

\section{Function Spaces}

Let $U$ be any open set in $\mathbb{R}^{n}$ and let $\sigma$ be the function:

$$
\sigma(x)=\left(1+|x|^{2}\right)^{1 / 2} .
$$

We shall define the following spaces of functions on $U$ with values in some given finite dimensional vector space $V$.

Definition 2.1. $C_{\delta}^{s}(U), s \in \mathbb{N}, \delta \in \mathbb{R}$, is the Banach space of functions $u$ on $U$ with values in $V$, of class $C^{s}$, such that:

$$
\|u\|_{C_{\delta}^{s}(U)}=\sup _{U}\left\{\sum_{|\alpha| \leqq s}\left|\sigma^{\delta+|\alpha|} D^{\alpha} u\right|\right\}<\infty .
$$

Definition 2.2. $H_{s, \delta}(U), s \in \mathbb{N}, \delta \in \mathbb{R}$, is the class of all functions $u$ on $U$ with values in $V$, possessing weak derivatives up to order $s$, such that $\sigma^{\delta+|\alpha|} D^{\alpha} u \in L^{2}(U)$ for each $|\alpha| \leqq s . H_{s, \delta}(U)$ is a Hilbert space with respect to the inner product:

$$
\left(u_{1}, u_{2}\right)_{H_{s, \delta}(U)}=\sum_{|\alpha| \leqq s}\left(\sigma^{\delta+|\alpha|} D^{\alpha} u_{1}, \sigma^{\delta+|\alpha|} D^{\alpha} u_{2}\right)_{L^{2}(U)} .
$$

We shall write the norm:

$$
\|u\|_{H_{s, \delta}(U)}=(u, u)_{H_{s, \delta}(U)}^{1 / 2} .
$$

As with the usual Sobolev spaces $H_{s}(U)$, these weighted Sobolev spaces $H_{s, \delta}(U)$ have the following density property:

Proposition 2.1. If $U$ has the segment property, the set of restrictions to $U$ of functions in $C_{0}^{\infty}\left(\mathbb{R}^{n}\right)$ is dense in $H_{s, \delta}(U)$ for each $s \in \mathbb{N}, \delta \in \mathbb{R}$.

Remark. The segment property (see [20]) says that the domain $U$ does not lie on both sides of its boundary.

Given $0<\varepsilon \leqq 1$, let $\varphi_{\varepsilon}$ be the differentiable transformation of $\mathbb{R}^{n}$ defined by:

$$
x \mapsto \varphi_{\varepsilon}(x)=\frac{x}{(\sigma(x))^{1-\varepsilon}}
$$


( $\varphi_{1}$ is the identity transformation of $\mathbb{R}^{n}$ ). Consider the operator $T_{\varepsilon}$, acting on functions $f$ on $\mathbb{R}^{n}$ with values in $V$ in the following way:

$$
T_{\varepsilon} f=f \circ \varphi_{\varepsilon}^{-1} .
$$

$T_{\varepsilon}$ is bijective, it is a linear operator, and satisfies $T_{\varepsilon}\left(f_{1} f_{2}\right)=T_{\varepsilon}\left(f_{1}\right) T_{\varepsilon}\left(f_{2}\right)$. Thus $T_{\varepsilon}$ is an automorphism of the ring of functions on $\mathbb{R}^{n}$ with values in $\mathbb{R}$. In [21] the following lemma is demonstrated:

Lemma 2.1. For each $0<\varepsilon \leqq 1, T_{\varepsilon}$ is an isomorphism:

and

a) $C_{\delta}^{s}(U) \rightarrow C_{\delta / \varepsilon}^{s}\left(\varphi_{\varepsilon}(U)\right)$

b) $H_{s, \delta}(U) \rightarrow H_{s,((\delta+n / 2) / \varepsilon)-n / 2}\left(\varphi_{\varepsilon}(U)\right)$.

The imbedding and multiplication theorems which follow were proven in [21] by using this lemma. We need to introduce:

Definition 2.3. An open subset $U \subset \mathbb{R}^{n}$ has the extended cone property if $\varphi_{\varepsilon}(U)$ has the cone property for each $0<\varepsilon \leqq 1$.

Remark. A domain $U$ is said to have the cone property (see [20]) if there exists a finite cone $C$ such that each point $x \in U$ is the vertex of a finite cone $C_{x}$ contained in $U$ and congruent to $C$ ( $C_{x}$ is obtained from $C$ by rigid motion). A domain satisfies the stronger requirement of Definition 2.3 if in addition it has a linear growth at infinity. This in particular excludes slabs contained between two parallel hyperplanes.

Theorem 2.1 (imbedding). If $U$ has the extended cone property, the following inclusion holds and is continuous :

$$
H_{s, \delta}(U) \subset C_{\delta^{\prime}}^{s^{\prime}}(U)
$$

if $s^{\prime}<s-n / 2, \delta^{\prime}<\delta+n / 2$.

Theorem 2.2 (multiplication). If $U$ has the extended cone property, then pointwise multiplication

$$
\left(f_{1}, f_{2}\right) \rightarrow f_{1} f_{2}
$$

is a continuous bilinear map:

$$
H_{s_{1}, \delta_{1}}(U) \times H_{s_{2}, \delta_{2}}(U) \rightarrow H_{s, \delta}(U)
$$

if $s_{1}, s_{2} \geqq s, s<s_{1}+s_{2}-n / 2, \delta<\delta_{1}+\delta_{2}+n / 2$.

Corollary. If $U$ has the extended cone property, then $H_{s, \delta}(U)$ is a Banach algebra if $s>n / 2, \delta>-n / 2$.

Remark. Theorems 2.1 and 2.2 are the strongest such results that can be obtained. For example, the functions which are the powers of $\sigma$ contradict any weakening of the conditions on $\delta$.

In addition to the imbedding and multiplication theorems, we shall need a composition theorem. We first show: 
Lemma 2.2. If $U$ has the extended cone property and $f$ is a diffeomorphism $U \rightarrow U^{\prime}$ such that $\operatorname{det}(D f) \geqq c>0$ and $f-\mathrm{id} \in H_{s+1, \delta-1}(U)$ with $s>n / 2, \delta>-n / 2$, then composition $u \rightarrow u \circ f$ is an isomorphism:

for every $\delta^{\prime} \in \mathbb{R}$.

$$
H_{0, \delta^{\prime}}\left(U^{\prime}\right) \rightarrow H_{0, \delta^{\prime}}(U)
$$

Proof. Let us denote

$$
y=f(x), x \in U .
$$

By the imbedding theorem there is a $\beta>0$ such that $f-\mathrm{id} \in C_{\beta-1}^{1}(U)$. It follows that there exist positive constants $c_{1}$ and $c_{2}$ such that:

$$
c_{1} \sigma(x) \leqq \sigma(y) \leqq c_{2} \sigma(x) \text {. }
$$

Considering the fact that

$$
d y=\operatorname{det}(D f) d x,
$$

we conclude that there exist positive constants $c_{1}^{\prime}$ and $c_{2}^{\prime}$ such that for every $u \in H_{0, \delta^{\prime}}\left(U^{\prime}\right)$ :

$$
c_{1}^{\prime} \int_{U}\left|\sigma(x)^{\delta^{\prime}} u \circ f(x)\right|^{2} d x \leqq \int_{U^{\prime}}\left|\sigma(y)^{\delta^{\prime}} u(y)\right|^{2} d y \leqq c_{2}^{\prime} \int_{U}\left|\sigma(x)^{\delta^{\prime}} u \circ f(x)\right|^{2} d x
$$

which shows the lemma.

Remark. If $U=\mathbb{R}^{n}$, the assumption $\operatorname{det}(d f) \geqq c>0$ is redundant, since $D f(x) \rightarrow I$ (the identity matrix) for $|x| \rightarrow \infty$ and $\operatorname{det}(D f) \neq 0$.

Lemma 2.3. If $U$ has the extended cone property and $f$ is a diffeomorphism $U \rightarrow U^{\prime}$ such that $\operatorname{det}(D f) \geqq c>0$ and $f-\mathrm{id} \in H_{s+1, \delta-1}(U)$ with $s>n / 2, \delta>-n / 2$, then $f^{-1}-\mathrm{id} \in H_{s+1, \delta-1}\left(U^{\prime}\right)$.

Proof. Applying Lemma 2.2 with $\delta^{\prime}=\delta-1$ to $u=f^{-1}$-id we obtain $f^{-1}$ $-\mathrm{id} \in H_{0, \delta-1}\left(U^{\prime}\right)$, since :

$$
\left(f^{-1}-\mathrm{id}\right) \circ f=-(f-\mathrm{id})
$$

We have:

$$
D\left(f^{-1}-\mathrm{id}\right)=\mathrm{h} \circ \mathrm{f}^{-1},
$$

where

$$
h=(D f)^{-1}-I \text {. }
$$

The facts that $D(f-\mathrm{id})$ belongs to $H_{s, \delta}(U)$, which is a Banach algebra by the corollary to the multiplication theorem, together with $\operatorname{det}(D f) \geqq c>0$ imply that $h \in H_{s, \delta}(U)$. Application of Lemma 2.2 with $\delta^{\prime}=\delta$ to $u=h \circ f^{-1}$ gives $D\left(f^{-1}-\mathrm{id}\right) \in H_{0, \delta}\left(U^{\prime}\right)$. For $2 \leqq k \leqq s+1$ we use the formula:

where

$$
D^{k}\left(f^{-1}-\mathrm{id}\right)=g_{k} \circ f^{-1}
$$

$$
g_{k}=((h+I) \cdot D)^{k-1} h
$$


The multiplication theorem implies that:

$$
g_{k} \in H_{s-k+1, \delta+k-1}(U) \text {. }
$$

Therefore, Lemma 2.2 with $\delta^{\prime}=\delta+k-1$ applied to $u=g_{k} \circ f^{-1}$ yields :

$$
D^{k}\left(f^{-1}-\mathrm{id}\right) \in H_{0, \delta+k-1}\left(U^{\prime}\right) .
$$

Consequently, we have $f^{-1}-\mathrm{id} \in H_{s+1, \delta-1}\left(U^{\prime}\right)$.

We shall now employ the above two lemmas to prove:

Theorem 2.3 (composition). If $U$ has the extended cone property and $f$ is a diffeomorphism $U \rightarrow U^{\prime}$ such that $\operatorname{det}(D f) \geqq c>0$ and $f-\mathrm{id} \in H_{s+1, \delta-1}(U)$ with $s>n / 2, \delta>-n / 2$, then composition $u \rightarrow u \circ f$ is an isomorphism:

$$
H_{s^{\prime}, \delta^{\prime}}\left(U^{\prime}\right) \rightarrow H_{s^{\prime}, \delta^{\prime}}(U)
$$

for every $s^{\prime} \leqq s+1, \delta^{\prime} \in \mathbb{R}$.

Proof. For $s^{\prime}=0$ the theorem reduces to Lemma 2.2. We first show by induction that $u \rightarrow u \circ f$ is a continuous map:

$$
H_{s^{\prime}, \delta^{\prime}}\left(U^{\prime}\right) \rightarrow H_{s^{\prime}, \delta^{\prime}}(U)
$$

for every $s^{\prime} \leqq s+1, \delta^{\prime} \in \mathbb{R}$. Indeed, let this statement be true for $s^{\prime}-1$ and every $\delta^{\prime} \in \mathbb{R}$ and let $u \in H_{s^{\prime}, \delta^{\prime}}\left(U^{\prime}\right)$. Then $D u \in H_{s^{\prime}-1, \delta^{\prime}+1}\left(U^{\prime}\right)$ and by the inductive hypothesis $(D u) \circ f \in H_{s^{\prime}-1, \delta^{\prime}+1}(U)$ and :

$$
\|(D u) \circ f\|_{H_{s^{\prime}-1, \delta^{\prime}+1}(U)} \leqq \text { const. }\|D u\|_{H_{s^{\prime}-1, \delta^{\prime}+1}\left(U^{\prime}\right)} .
$$

We have:

$$
D(u \circ f)=D f \cdot(D u) \circ f .
$$

Since $D(f-\mathrm{id}) \in H_{s, \delta}(U)$ and by Theorem 2.2 pointwise multiplication is a continuous bilinear map:

$$
H_{s, \delta}(U) \times H_{s^{\prime}-1, \delta^{\prime}+1}(U) \rightarrow H_{s^{\prime}-1, \delta^{\prime}+1}(U),
$$

we conclude that $D(u \circ f) \in H_{s^{\prime}-1, \delta^{\prime}+1}(U)$ and:

$$
\|D(u \circ f)\|_{H_{s^{\prime}-1, \delta^{\prime}+1}(U)} \leqq \text { const. }\|u\|_{H_{s^{\prime}, \delta^{\prime}}\left(U^{\prime}\right)} .
$$

On the other hand the inductive hypothesis gives also $u \circ f \in H_{s^{\prime}-1, \delta^{\prime}}(U)$ and:

$$
\|u \circ f\|_{H_{s^{\prime}-1, \delta^{\prime}}(U)} \leqq \text { const. }\|u\|_{H_{s^{\prime}-1, \delta^{\prime}}\left(U^{\prime}\right)} .
$$

It follows that $u \circ f \in H_{s^{\prime}, \delta^{\prime}}(U)$ and:

$$
\|u \circ f\|_{H_{s^{\prime}, \delta^{\prime}}(U)} \leqq \text { const. }\|u\|_{H_{s^{\prime}, \delta^{\prime}}\left(U^{\prime}\right)},
$$

which completes the inductive step.

Since by Lemma $2.3 f^{-1}-\mathrm{id} \in H_{s+1, \delta-1}\left(U^{\prime}\right)$, the continuity of the inverse of $u \mapsto u \circ f$ follows as above, in view of the fact that by (2.1) and the condition $\operatorname{det}(D f)$ $\geqq c>0$ also $U^{\prime}$ enjoys the extended cone property.

Let now $D_{s+1, \delta-1}\left(\mathbb{R}^{n}\right)$ denote the set of all differentiable transformations $f$ of $\mathbb{R}^{n}$ such that $f-\mathrm{id} \in H_{s+1, \delta-1}\left(\mathbb{R}^{n}\right)$. In view of Theorem 2.3 we have: 
Corollary. If $s>n / 2$ and $\delta>-n / 2$, then $D_{s+1, \delta-1}\left(\mathbb{R}^{n}\right)$ is a topological group with respect to composition.

Denoting $\bar{x}=\left(x^{1}, \ldots, x^{n-1}\right)$ and $\bar{\sigma}(\bar{x})=\left(1+|\bar{x}|^{2}\right)^{1 / 2}$, let $\Omega_{\theta}$ be the domain defined by:

$$
\Omega_{\theta}=\left\{x \in \mathbb{R}^{n} \mid \frac{\left|x^{0}\right|}{\bar{\sigma}(\bar{x})}<\theta\right\}
$$

where $0<\theta<1$. This domain will play an important role in the sections concerning the evolution problem. It enjoys the extended cone property. For such a domain, replacing $\sigma$ by $\bar{\sigma}$ in Definitions 2.1 and 2.2 gives equivalent norms.

We define on $\Omega_{\theta}$ the function

$$
\tau(x)=\frac{x^{0}}{\bar{\sigma}(\bar{x})} .
$$

This function induces a foliation of $\Omega_{\theta}$ :

$$
\left.\Omega_{\theta}=\bigcup_{\tau \in I_{\theta}} \Sigma_{\tau}, I_{\theta}=\right]-\theta, \theta[,
$$

where $\Sigma_{\tau}$ are the level surfaces of $\tau$. The following map is a diffeomorphism:

$$
\begin{gathered}
\psi: \Omega_{\theta} \rightarrow I_{\theta} \times \mathbb{R}^{n-1} \\
\text { by }: x \rightarrow(\tau, \bar{x}) .
\end{gathered}
$$

Let $G$ be the operator which sends functions $u$ defined on $\Omega_{\theta}$ with values in some given finite dimensional vector space $V$ into functions $G u$ defined on $I_{\theta} \times \mathbb{R}^{n-1}$ with values in $V$ by:

$$
G u=u \circ \psi^{-1} .
$$

Taking into account the fact that the euclidean measure $d \tau d \bar{x}$ on $I_{\theta} \times \mathbb{R}^{n-1}$ is related to the euclidean measure $d x$ on $\Omega_{\theta}$ by:

$$
d x=\bar{\sigma}(\bar{x}) d \tau d \bar{x},
$$

one can show that there exist positive constants $c_{1}$ and $c_{2}$ such that for every $u \in H_{s, \delta}\left(\Omega_{\theta}\right)$

$$
\begin{gathered}
c_{1}\|u\|_{H_{s, \delta}\left(\Omega_{\theta}\right)}^{2} \leqq \int_{I_{\theta} \times \mathbb{R}^{n-1}} \sum_{k+|\beta| \leqq s}\left|\bar{\sigma}^{\delta+\frac{1}{2}+|\beta|} D_{\tau}^{k} \bar{D}^{\beta} G u\right|^{2} d \tau d \bar{x} \\
\leqq c_{2}\|u\|_{H_{s, \delta}\left(\Omega_{\theta}\right)}^{2} .
\end{gathered}
$$

Let us define the following restriction norm to the hypersurfaces $\Sigma_{\tau}$ :

$$
\|u\|_{H_{s, \delta}\left(\Sigma_{\tau}, \Omega_{\theta}\right)}=\left(\sum_{k=0}^{s}\left\|\left.D_{0}^{k} u\right|_{\Sigma_{\tau}}\right\|_{H_{s-k, \delta+k}\left(\mathbb{R}^{n-1)}\right.}\right)^{1 / 2}
$$

The following restriction lemma was proven in [22] by employing the isomorphism (2.5):

Lemma 2.4 (restriction). For each $\tau \in I_{\theta}$, the following inclusion holds and is continuous:

$$
H_{s+1, \delta}\left(\Omega_{\theta}\right) \subset H_{s, \delta+\frac{1}{2}}\left(\Sigma_{\tau}, \Omega_{\theta}\right)
$$

for every $s \in \mathbb{N}, \delta \in \mathbb{R}$. 


\section{The Constraint Equations}

Initial data for the vacuum Einstein equations consist of giving a riemannian metric $g$ and a 2-covariant symmetric tensorfield $k$ on the hypersurface $x^{0}=0$ which we call $\Sigma$. These are going to be respectively the first and second fundamental forms of $\Sigma$ in the spacetime $(\Omega, \gamma)$ to be constructed. It follows from the Einstein equations that a necessary condition for the existence of such a spacetime is that the initial data satisfy the constraints (equivalent to $G_{\mid \Sigma}^{0 \mu}=0$ ):

$$
\begin{gathered}
\delta_{g}\left(k-\left(\operatorname{tr}_{g} k\right) g\right)=0 \\
R(g)-|k|_{g}^{2}+\left(\operatorname{tr}_{g} k\right)^{2}=0,
\end{gathered}
$$

where $R(g)$ and $\delta_{g}$ are respectively the scalar curvature and divergence operator of the metric $g$. We shall assume for simplicity that $\Sigma$ is a maximal spacelike hypersurface of $(\Omega, \gamma)$. Then the constraints reduce to:

$$
\begin{aligned}
\operatorname{tr}_{g} k=\delta_{g} k & =0 \\
R(g)-|k|_{g}^{2} & =0 .
\end{aligned}
$$

In York's formulation of the initial value problem $[9,10]$, one gives on $\Sigma$ a riemannian metric $\tilde{g}$ and a 2 -covariant symmetric tensorfield $\tilde{k}$ subject to the conditions

$$
\operatorname{tr}_{\tilde{g}} \tilde{k}=\delta_{\tilde{g}} \tilde{k}=0 .
$$

Then if one can find a positive function $\phi$ satisfying the Lichnerowicz equation:

$$
\Delta_{\tilde{g}} \phi-\frac{1}{8} R(\tilde{g}) \phi+\frac{1}{8}|\tilde{k}|_{\tilde{g}}^{2} \phi^{-7}=0,
$$

the riemannian metric $g=\phi^{4} \tilde{g}$, together with $k=\phi^{-2} \tilde{k}$ satisfy (3.1) and (3.2). We shall therefore study the space of solutions to (3.3) and the existence of positive solutions to (3.4).

We shall use theorems on linear elliptic systems on $\mathbb{R}^{n}$ which were proven in [21] in the more general context of euclidean at infinity manifolds. These results are extensions of results obtained by Cantor [23]. Let $L$ be a linear differential operator of order $m$ on $\mathbb{R}^{n}$ :

$$
L u=\sum_{k=0}^{m} a_{k} \cdot D^{k} u,
$$

where $u$ and $L u$ are $\mathbb{R}^{n}$ valued functions on $\mathbb{R}^{n}$. We introduce the following hypotheses:

Hypothesis I (ellipticity). At each $x \in \mathbb{R}^{n}$ and for every vector $\xi \neq 0$ we have:

$$
\operatorname{det}\left(a_{m}(x) \cdot \xi^{m}\right)>0,
$$

where $a_{m}(x) \cdot \xi^{m}$ is the matrix :

$$
\begin{gathered}
\left(a_{m}(x) \cdot \xi^{m}\right)_{J}^{I}=a_{i_{1} \ldots i_{m}} J(x) \xi^{i_{1} \ldots \xi^{i_{m}}} \\
I, J=1, \ldots, N .
\end{gathered}
$$

Remark. The ellipticity condition implies that $\mathrm{Nm}$ is even. 
Hypothesis II (regularity). There exist nonnegative integers $s_{k}$ and real numbers $\delta_{k}, 0 \leqq k \leqq m$, such that:

$$
s_{k}>\frac{n}{2}+k-m+1, \delta_{k}>m-k-\frac{n}{2}: 0 \leqq k \leqq m
$$

and

where

1) $a_{k} \in H_{s_{k}, \delta_{k}}: 0 \leqq k \leqq m-1$

2) $a_{m}-A_{m} \in H_{s_{m}, \delta_{m}}$,

$$
L_{\infty} u=A_{m} \cdot D^{m} u
$$

is a homogeneous elliptic (hypothesis I) operator of order $m$ with constant coefficients.

Remark. By virtue of hypotheses I and II there exists a positive real number $\lambda$, called the ellipticity constant, such that for every $x \in \mathbb{R}^{n}$ and every vector $\xi$ :

$$
\operatorname{det}\left(a_{m}(x) \cdot \xi^{m}\right) \geqq \lambda \mid \xi^{N m}
$$

The following theorems were proven in [21]:

Theorem 3.1. The homogeneous elliptic operator with constant coefficients on $\mathbb{R}^{n}$ :

$$
L_{\infty}=A_{m} \cdot D^{m}
$$

of order $m<n$, is an isomorphism:

$$
H_{s, \delta} \rightarrow H_{s-m, \delta+m}
$$

if $s \geqq m$ and $-n / 2<\delta<-m+n / 2$.

Remark. Theorem 3.1 in the special case of the Laplace operator of the euclidean metric is due to McOwen [24].

Let us denote by $E$ the set of linear differential operators of order $m$ on $\mathbb{R}^{n}$ which satisfy hypotheses I and II. We define a metric (hence a topology) on $E$ by setting:

$$
d\left(L, L^{\prime}\right)=\max _{0 \leqq k \leqq m}\left\|a_{k}-a_{k}^{\prime}\right\|_{H_{s_{k}, \delta_{k}}} .
$$

We set:

$$
s^{\prime}=\min _{0 \leqq k \leqq m}\left\{s_{k}\right\}+m .
$$

Theorem 3.2. Let $L$ be a linear differential operator on $\mathbb{R}^{n}$ of order $m<n$, such that $L$ belongs to a continuous (in the sense of $d$ ) curve $\left\{L_{t} \mid t \in I \equiv[0,1]\right\}, L_{1}=L$, of injective operators in $E$. Then if $L_{0}$ is an isomorphism:

$$
H_{s, \delta} \rightarrow H_{s-m, \delta+m}
$$

with $m \leqq s \leqq s^{\prime}$ and $-n / 2<\delta<-m+n / 2$, the same is true for $L$.

In applying the above theorems we shall make use of the following proposition: 
Proposition 3.1. If $L$ is a differential operator on $\mathbb{R}^{n}$, or order $m<n$, satisfying hypotheses $I$ and $I I$, then every $u \in \operatorname{ker}(L)$ which belongs to $H_{s, \delta}$ with $m \leqq s \leqq s^{\prime}$ and $\delta>-n / 2$, belongs also to $H_{s, \delta^{\prime}}$ for any $\delta^{\prime}<-m+n / 2$.

Proof. By hypothesis II, $a_{k} \in H_{s_{k}, \delta_{k}}: 0 \leqq k \leqq m-1$ and $a_{m}-A_{m} \in H_{s_{m}, \delta_{m}}$ with $\delta_{k}>m$ $-k-n / 2$ for each $0 \leqq \mathrm{k} \leqq \mathrm{m}$. Therefore:

$$
\varepsilon \equiv \min _{0 \leqq k \leqq m}\left\{\delta_{k}-m+k+n / 2\right\}>0 .
$$

We shall show that every $u \in \operatorname{ker}(L)$ which belongs to $H_{s, \delta}, \delta>-n / 2$, belongs also to $H_{s, \delta}$ for any $\delta^{\prime}<\min \{\delta+\varepsilon,-m+n / 2\}$. From this result the proposition follows by induction. Let us define:

$$
f \equiv\left(L_{\infty}-L\right) u=-\sum_{k=0}^{m-1} a_{k} \cdot D^{k} u-\left(a_{m}-A_{m}\right) \cdot D^{m} u .
$$

By applying the multiplication theorem we find: $f \in H_{s-m, \delta^{\prime}+m}$ for any $\delta^{\prime}<\delta+\varepsilon$. On the other hand, since $u \in \operatorname{ker}(L)$ we have:

$$
L_{\infty} u=f \text {, }
$$

which by Theorem 3.1 implies $u \in H_{s, \delta^{\prime}}$ if $\delta^{\prime}<-\mathrm{m}+n / 2$ also.

Remark. The condition on $\delta$ in Theorems 3.1 and 3.2 is exactly the one that would be expected from standard results for Poisson's equation $\Delta \phi=\varrho$ in 3 dimensions. The lower bound on $\delta,-3 / 2<\delta$, by the imbedding theorem implies that $\phi \rightarrow 0$ at infinity. The upper bound, $\delta<-1 / 2$, on the other hand, says that in general $\phi$ does not fall off at infinity faster than $1 / r$.

We shall apply the above theorems to the problem of the initial value constraints. First we need to show how to obtain solutions to (3.3), namely how to obtain 2-covariant symmetric tensorfields on $\left(\mathbb{R}^{n}, \tilde{g}\right)$ which are transverse and traceless. This is done in the York decomposition theorem which follows (Theorem 3.4). A previous version of this theorem is given in [12]. However the proof given there fails (except if the restriction is imposed that $\tilde{g}$ is everywhere close to e) because it relies on Lemma 5.5.2 of [23] which is false. Our proof of the theorem employs the proposition that if $\tilde{g}$ is asymptotically euclidean it admits no nontrivial conformal killing vectorfields tending to zero at infinity. This proposition follows from:

Theorem 3.3. Let $u$ be a $\mathbb{R}^{N}$ valued function on $\mathbb{R}^{n}$, of class $C^{m+1}$ satisfying the following condition: The derivatives of $u$ of order $m+1$ can be expressed as a linear combination, with $C^{0}$ coefficients, of the derivatives of order $\leqq m$ :

$$
D^{m+1} u=\sum_{k=0}^{m} b_{k} \cdot D^{k} u \text {. }
$$

If $b_{k} \in C_{\delta_{k}}^{0}$ with:

$$
\delta_{k}>m+1-k: 0 \leqq k \leqq m
$$

and $u \in C_{\beta}^{m}, \beta>0$, then $u=0$ on $\mathbb{R}^{n}$. 
Proof. Let $B_{R}$ denote the open ball of radius $R$ in $\mathbb{R}^{n}$. We have the following calculus inequality: there exists a constant $c$ independent of $R$ such that for every $u \in C_{\beta}^{m+1}\left(\mathbb{R}^{n}\right), \beta>0$ :

$$
\|u\|_{C_{\beta}^{m}\left(\mathbb{R}^{n}-\bar{B}_{R}\right)} \leqq c\left\|D^{m+1} u\right\|_{C_{\beta+m+1}^{0}\left(\mathbb{R}^{n}-\bar{B}_{R}\right)} .
$$

On the other hand, if $u$ satisfies conditon (3.5) we have:

$$
\left\|D^{m+1} u\right\|_{C_{\beta+m+1}^{0}\left(\mathbb{R}^{n}-\bar{B}_{R}\right)} \leqq \mu(R)\|u\|_{C_{\beta}^{m}\left(\mathbb{R}^{n}-\bar{B}_{R}\right)},
$$

where:

$$
\mu(R)=\sum_{k=0}^{m}\left\|b_{k}\right\|_{C_{m+1-k}^{0}\left(\mathbb{R}^{n}-\bar{B}_{R}\right)} .
$$

Denoting:

$$
B=\sum_{k=0}^{m}\left\|b_{k}\right\|_{C_{\delta_{k}}^{0}}
$$

and :

$$
\varepsilon=\min _{0 \leqq k \leqq m}\left\{\delta_{k}-m-1+k\right\},
$$

we have $\varepsilon>0$ and:

$$
\mu(R) \leqq R^{-\varepsilon} B .
$$

Inequalities (3.6), (3.7) and (3.8) give:

$$
\|u\|_{C_{\beta}^{m}\left(\mathbb{R}^{n}-\bar{B}_{R}\right)} \leqq c B R^{-\varepsilon}\|u\|_{C_{\beta}^{m}\left(\mathbb{R}^{n}-\bar{B}_{R}\right)},
$$

which, if:

$$
R>(c B)^{1 / \varepsilon}
$$

implies $u=0$ on $\mathbb{R}^{n}-\bar{B}_{R}$.

Consider now an $\mathbb{R}^{n}$ valued function $u$ on $\mathbb{R}^{n}$, of class $C^{m+1}$, such that:

$$
D^{k} u\left(x_{0}\right)=0: 0 \leqq k \leqq m .
$$

Let $B\left(x_{0}, r\right)$ be the open ball of radius $r \leqq 1$ in $\mathbb{R}^{n}$, centered at $x_{0}$. Taylor's theorem gives:

$$
\|u\|_{C_{b}^{m}\left(B\left(x_{0}, r\right)\right)} \leqq c^{\prime} r\left\|D^{m+1} u\right\|_{C_{b}^{0}\left(B\left(x_{0}, r\right)\right)} .
$$

On the other hand, if $u$ satisfies condition (3.5) we have:

$$
\left\|D^{m+1} u\right\|_{C_{b}^{0}\left(B\left(x_{0}, r\right)\right)} \leqq B^{\prime}\|u\|_{C_{b}^{\prime \prime}\left(B\left(x_{0}, r\right)\right)},
$$

where:

$$
B^{\prime}=\sum_{k=0}^{m}\left\|b_{k}\right\|_{C_{b}^{0}\left(B\left(x_{0}, 1\right)\right)} .
$$

Inequalities (3.9) and (3.10) give:

$$
\|u\|_{C_{b}^{m}\left(B\left(x_{0}, r\right)\right)} \leqq c^{\prime} B^{\prime} r\|u\|_{C_{b}^{m}\left(B\left(x_{0}, r\right)\right)},
$$


which, if:

$$
r<1 / c^{\prime} B^{\prime}
$$

implies $u=0$ on $B\left(x_{0}, r\right)$.

Let us denote:

$$
U=\left\{x \in \mathbb{R}^{n} \mid D^{k} u(x)=0: 0 \leqq k \leqq m\right\} .
$$

By the proceeding argument this set is open, Since $D^{k} u: 0 \leqq k \leqq m$ is continuous, $U$ is closed as well. Furthermore $U$ is non-empty since we have shown that $u=0$ on $\mathbb{R}^{n}-\bar{B}_{R}$. Thus $U=\mathbb{R}^{n}$ and $u=0$ on $\mathbb{R}^{n}$.

As a corollary of the above theorem we have:

Proposition 3.2. Let $g$ be a riemannian metric on $\mathbb{R}^{n}$ such that $g-e \in C_{\beta}^{3}, \beta>0$. If $X$ is a conformal killing vectorfield of $g$ of class $C^{3}$ such that $X \in C_{\beta}^{2}$, then $X=0$ on $\mathbb{R}^{n}$.

Proof. If $X$ is a conformal killing vectorfield of $g$, one can express the 3 rd covariant derivatives of $X$ with respect to $g$ in the form:

$$
\nabla_{g}^{3} X=R_{0} \cdot \nabla_{g} X+R_{1} \cdot X
$$

where $R_{0}$ and $R_{1}$ are linear expressions in $\operatorname{Riem}(g)$ and $\nabla_{g} \operatorname{Riem}(g)$ respectively. Hence Theorem 3.3 applies, taking $m=2$.

Remark. An analogous proposition for killing vectorfields and $n=3$ but more general riemannian manifolds has been proven by Choquet and Choquet-Bruhat in [25].

Let us denote by $L_{g}$ the conformal killing operator of $g$, namely the $1^{\text {st }}$ order linear differential operator which maps vectorfields $X$ into 2-covariant symmetric tensorfields :

$$
L_{g}(X)=L_{X} g+\frac{2}{n}\left(\delta_{g} X\right) g .
$$

Consider the $2^{\text {nd }}$ order linear differential operator $\delta_{g} \circ L_{g}$ which maps vectorfields into vectorfields. We shall show:

Lemma 3.1. Let $n>2, s>n / 2+3,-n / 2<\delta<-2+n / 2$. If $g$ is a riemannian metric on $\mathbb{R}^{n}$ such that $g-e \in H_{s, \delta}$, then the operator $\delta_{g} \circ L_{g}$ is an isomorphism:

$$
H_{s, \delta} \rightarrow H_{s-2, \delta+2} \text {. }
$$

Proof. The operator $\delta_{g} \circ L_{g}$ satisfies hypotheses I and II. We shall first show that it is injective. Let $X \in \operatorname{ker}\left(\delta_{g} \circ L_{g}\right)$ and $X \in H_{2, \delta}$ with $\delta>-n / 2$. By Proposition 3.1, $X$ belongs also to $H_{2,-1}$. Integrating the equation

$$
g\left(X, \delta_{g} \circ L_{g}(X)\right)=0
$$

over $\mathbb{R}^{n}$ with the canonical measure $d \mu_{g}$ of the riemannian metric $g$ we obtain:

$$
\int_{\mathbb{R}^{n}}\left|L_{g}(X)\right|_{g}^{2} d \mu_{g}=0
$$

This result is obtained by approximating $X$ with vectorfields $X_{n} \in C_{0}^{\infty}$ (Proposition 2.1) and holds because $X \in H_{2,-1}$. It follows that $L_{g}(X)=0$, hence $X$ is a conformal 
killing vectorfield of $g$. If now $X \in H_{s, \delta}$ with $s>n / 2+3, \delta>-n / 2$, the imbedding theorem implies that $X \in C_{\beta}^{3}$ with $\beta>0$. Therefore Proposition 3.2 applies and $X=0$ on $\mathbb{R}^{n}$.

Thus $\delta_{g} \circ L_{g}$ is injective on $H_{s, \delta}$ for any riemannian metric $g$ satisfying the hypotheses of the lemma. Let now:

$$
g_{t}=e(1-t)+t g, t \in I \equiv[0,1]
$$

and consider the family of operators: $\left\{\delta_{g_{t}} \circ L_{g_{t}} \mid t \in I\right\}$. This family is a continuous curve of injective operators in $E$ and

$$
\delta_{g_{0}} L_{g_{0}}=\delta_{e^{\circ}} L_{e}
$$

is an isomorphism: $H_{s, \delta} \rightarrow H_{s-2, \delta+2}$ by Theorem 3.1. Consequently, the hypotheses of Theorem 3.2 are satisfied for the operator $\delta_{g} \circ L_{g}$ and the lemma follows.

We are now in position to prove:

Theorem 3.4 (York decomposition). Let $n>2, s>n / 2+3,-n / 2<\delta<-2+n / 2$ and let $g$ be a riemannian metric on $\mathbb{R}^{n}$ such that $g-e \in H_{s, \delta}$. Then the space of 2-covariant tensorfields on $\left(\mathbb{R}^{n}, g\right)$ of class $H_{s-1, \delta+1}$ decomposes into:

$$
H_{s-1, \delta+1}=\operatorname{tr}_{g}\left(H_{s-1, \delta+1}\right) g \oplus L_{g}\left(H_{s, \delta}\right) \oplus J_{s-1, \delta+1},
$$

where:

$$
J_{s-1, \delta+1}=\left\{h \in H_{s-1, \delta+1} \mid \operatorname{tr}_{g} h=\delta_{g} h=0\right\} .
$$

Proof. We first decompose:

$$
H_{s-1, \delta+1}=\operatorname{tr}_{g}\left(H_{s-1, \delta+1}\right) g \oplus G_{s-1, \delta+1},
$$

where:

$$
G_{s-1, \delta+1}=\left\{h \in H_{s-1, \delta+1} \mid \operatorname{tr}_{g} h=0\right\} .
$$

Decomposition (3.12) follows from the fact that $g-e$ belongs to a Banach algebra (multiplication theorem) and we have $\operatorname{det} g \geqq c>0$ (since by the imbedding theorem $g-e \in C_{\beta}^{0}$ for some $\beta>0$ ). It remains to be shown that $G_{s-1, \delta+1}$ decomposes into:

$$
G_{s-1, \delta+1}=L_{g}\left(H_{s, \delta}\right) \oplus J_{s-1, \delta+1} .
$$

For all vector fields $X \in H_{s, \delta}$ it holds: $\operatorname{tr}_{g}\left(L_{g}(X)\right)=0$. Hence we have:

$$
L_{g}\left(H_{s, \delta}\right) \subset G_{s-1, \delta+1} \text {. }
$$

On the other hand we have also:

$$
J_{s-1, \delta+1}=\left\{h \in G_{s-1, \delta+1} \mid \delta_{g} h=0\right\} .
$$

By Lemma 3.1 the composition $\delta_{g} \circ L_{g}$ is an isomorphism: $H_{s, \delta} \rightarrow H_{s-2, \delta+2}$. Therefore, the decomposition (3.13) follows.

The above theorem shows how to construct solutions to (3.3). Given a riemannian metric $g$ on $\mathbb{R}^{n}, n>2$, satisfying the hypotheses of the theorem, and given any 2-covariant symmetric tensorfield $h$ on $\mathbb{R}^{n}$ belonging to the class 
$H_{s-1, \delta+1}$, then we can find a vectorfield $X \in H_{s, \delta}$ such that

$$
k \equiv h-\frac{1}{n}\left(\operatorname{tr}_{g} h\right) g-L_{g}(X)
$$

is a 2-covariant symmetric tensorfield of class $H_{s-1, \delta+1}$ satisfying:

$$
\operatorname{tr}_{g} k=\delta_{g} k=0 \text {. }
$$

We now turn our attention to the nonlinear equation (3.4). We shall need:

Theorem 3.5. Let $g$ be a riemannian metric anf $f$ a nonnegative function on $\mathbb{R}^{n}, n>2$, such that $g-e \in H_{s^{\prime}, \delta^{\prime}}$ and $f \in H_{s^{\prime}-2, \delta^{\prime}+2}$ with: $s^{\prime}>n / 2+1, \delta^{\prime}>-n / 2$. Then the operator $\Delta_{g}-f$ (acting on scalar functions on $\mathbb{R}^{n}$ ) is an isomorphism:

$$
H_{s, \delta} \rightarrow H_{s-2, \delta+2}
$$

for each $2 \leqq s \leqq s^{\prime},-n / 2<\delta<-2+n / 2$.

Proof. The operator $\Delta_{g}-f$ satisfies hypotheses I and II. We shall first show that is injective. Let $u \in \operatorname{ker}\left(\Delta_{g}-f\right)$ and $u \in H_{2, \delta}$ with $\delta>-n / 2$. By Proposition 3.1, $u$ belongs also to $H_{2,-1}$. Integrating the equation

$$
u\left(\Delta_{g}-f\right) u=0
$$

over $\mathbb{R}^{n}$ with the canonical measure $d \mu_{g}$ of the riemannian metric $g$ we obtain:

$$
\int_{\mathbb{R}^{n}}\left\{|D u|_{g}^{2}+f u^{2}\right\} d \mu_{g}=0
$$

(a result justified by taking a sequence $\left\{u_{n}\right\} \subset C_{0}^{\infty}$ such that $u_{n} \rightarrow u \in H_{2,-1}$ ). Thus $u=0$ on $\mathbb{R}^{n}$. We conclude that the operator $\Delta_{g}-f$ is injective on $H_{s, \delta}$ for any riemannian metric $g$ and nonnegative function $f$ satisfying the hypotheses of the theorem. Setting:

$$
g_{t}=e(1-t)+t g, \quad f_{t}=t f, \quad t \in I \equiv[0,1],
$$

the family $\left\{A_{t} \equiv \Delta_{g_{t}}-f_{t} \mid t \in I\right\}$ is a continuous curve of injective operators in $E$ and

$$
A_{0}=\Delta_{e}
$$

is an isomorphism: $H_{s, \delta} \rightarrow H_{s-2, \delta+2}$ by Theorem 3.1. Thus, the hypotheses of Theorem 3.2 are satisfied for the operator $\Delta_{g}-f=A_{1}$ and the theorem follows.

In the following we shall employ the above theorem to show that the Lichnerowicz equation (3.4) has one and only one positive solution $\phi$ tending to 1 at infinity, if $\tilde{g}$ is asymptotically euclidean, $R(\tilde{g}) \geqq 0$ and $\tilde{k}$ tends to 0 at infinity. We do this in two steps, following the approach of Cantor [13].

Lemma 3.2. Let $g$ be a riemannian metric on $\mathbb{R}^{n}, n>2$, such that $g-e \in H_{s, \delta}$ with $s>n / 2+2,-n / 2<\delta<-2+n / 2$ and $R(g) \geqq 0$. Then there exists a unique riemannian metric $g^{\prime}$, conformally equivalent to $g$, such that $g^{\prime}-e \in H_{s, \delta}$ and $R\left(g^{\prime}\right)=0$.

Proof. Setting:

$$
g^{\prime}=\chi^{4^{\prime} /(n-2)} g, \chi>0
$$


we find:

$$
\chi^{(n+2) /(n-2)} R\left(g^{\prime}\right)=\chi R(g)-4\left(\frac{n-1}{n-2}\right) \Delta_{g} \chi .
$$

Therefore the problem reduces to showing that the differential equation

$$
\Delta_{g} \chi-\frac{1}{4}\left(\frac{\mathrm{n}-2}{\mathrm{n}-1}\right) \mathrm{R}(\mathrm{g}) \chi=0
$$

has a unique positive solution $\chi$, such that $\chi-1 \in H_{s, \delta}$. Setting $\bar{\chi}=\chi-1$, we have:

$$
\left(\Delta_{g}-\frac{1}{4}\left(\frac{n-2}{n-1}\right) R(g)\right) \bar{\chi}=\frac{1}{4}\left(\frac{n-2}{n-1}\right) R(g) .
$$

The differential operator on the left satisfies the hypotheses of Theorem 3.5 (with $s^{\prime}=s^{\prime}, \delta=\delta^{\prime}$ ) and the right hand side belongs to $H_{s-2, \delta+2}$. Consequently there exists a unique $\bar{\chi} \in H_{s, \delta}$ satisfying (3.17). Since $\chi=1+\bar{\chi} \in C^{2}$ (imbedding theorem) and $R(g) \geqq 0$, equation (3.16) satisfies the strong maximum principle ([26]): $\chi$ does not attain on $\mathbb{R}^{n}$ a nonpositive minimum or a nonnegative maximum unless $\chi=0$ everywhere. However $\chi \rightarrow 1$ for $|x| \rightarrow \infty$ (imbedding theorem). We conclude:

$$
0<c \leqq \chi \leqq 1 .
$$

Following the argument of Cantor in [13] and employing in place of his Theorem 1.5 the extension given by Theorem 3.5 we can show:

Lemma 3.3. Let $g$ be a riemannian metric and $M$ a nonnegative function on $\mathbb{R}^{3}$ such that $g-e \in H_{s, \delta}$ and $M \in H_{s-2, \delta+2}$ with $s \geqq 4,-3 / 2<\delta<-1 / 2$. Then the semilinear elliptic differential equation

$$
\Delta_{g} \psi+M \psi^{-7}=0
$$

has one and only one positive solution $\psi$ such that $\psi-1 \in H_{s, \delta}$. Furthermore, $\psi \geqq 1$.

Combining the above two lemmas we obtain the following theorem on existence and uniqueness of positive solutions to the Lichnerowicz equation:

Theorem 3.6. Let $s \geqq 4,-3 / 2<\delta<-1 / 2$ and let $\tilde{g}$ be a riemannian metric and $\tilde{k} a$ 2-covariant symmetric tensorfield on $\mathbb{R}^{3}$ such that $\tilde{g}-e \in H_{s, \delta}, \tilde{k} \in H_{s-1, \delta+1}$ and $R(\tilde{g})$ $\geqq 0$. Then the Lichnerowicz equation (3.4) has one and only one positive solution $\phi$ such that $\phi-1 \in H_{s, \delta}$.

Proof. By Lemma 3.2 there exists a unique riemannian metric $g^{\prime}$, conformally equivalent to $\tilde{g}$ :

$$
g^{\prime}=\chi^{4} \tilde{g},
$$

such that $g^{\prime}-e \in H_{s, \delta}$ and $R\left(g^{\prime}\right)=0$. Setting then:

$$
k^{\prime}=\chi^{-2} \tilde{k}
$$

there exists according to Lemma 3.3 a unique positive solution $\psi$ to the equation:

$$
\Delta_{g^{\prime}} \psi+\frac{1}{8}\left|k^{\prime}\right|_{g^{\prime}}^{2} \psi^{-7}=0
$$


such that $\psi-1 \in H_{s, \delta}$. Thus, taking into account the fact that:

$$
\Delta_{g^{\prime}} \chi=\chi^{-5}\left(\Delta_{\tilde{g}}-\frac{1}{8} R(\tilde{g})\right) \chi
$$

we conclude that $\phi \equiv \chi \psi$ is the unique positive solution of:

$$
\Delta_{\tilde{g}} \phi-\frac{1}{8} R(\tilde{g}) \phi+\frac{1}{8}|\tilde{k}|_{\tilde{g}}^{2} \phi^{-7}=0,
$$

such that $\phi-1 \in H_{s, \delta}$.

We conclude that the riemannian metric $g=\phi^{4} \tilde{g}$ together with $k=\phi^{-2} \tilde{k}$ are such that $(g-e, k) \in H_{s, \delta} \times H_{s-1, \delta+1}$ and satisfy the nonlinear constraint (3.2).

\section{Harmonic Coordinates}

The vacuum Einstein equations $R^{\mu v}=0$ are a degenerate differential system. This is of course due to their geometric nature: if $(\Omega, \gamma)$ is a solution and $f$ is a diffeomorphism: $\Omega \rightarrow \Omega^{\prime}$, then $\left(\Omega^{\prime}, \mathrm{f}_{*} \gamma\right)$ is also a solution. One viable method of reducing the Einstein equations to a hyperbolic system is by means of the harmonic coordinate condition:

$$
\square_{\gamma} x^{\mu}=0 \text {. }
$$

An algebraic computation shows that:

$$
R^{\mu \nu}=R_{h}^{\mu \nu}+\frac{1}{2}\left(\gamma^{\mu \alpha} D_{\alpha} \Gamma^{\nu}+\gamma^{\nu \alpha} D_{\alpha} \Gamma^{\mu}\right)
$$

where:

$$
\Gamma^{\mu}=\gamma^{\alpha \beta} \Gamma_{\alpha \beta}^{\mu}=-\square_{\gamma} x^{\mu}
$$

and :

$$
R^{\mu v}=\frac{1}{2}\left\{\gamma^{\alpha \beta} D_{\alpha} D_{\beta} \gamma^{\mu v}-B^{\mu v}\left(\gamma^{\alpha \beta}, D_{\varrho} \gamma^{\alpha \beta}\right)\right\} .
$$

Here we have defined:

$$
B^{\mu \nu}=P_{\alpha \beta, \kappa \lambda}^{\mu \nu, \varrho \sigma} D_{\varrho} \gamma^{\alpha \beta} D_{\sigma} \gamma^{\kappa \lambda},
$$

$P$ being a rational function of $\gamma^{\alpha \beta}$, with numerator a homogeneous polynomial of degree 8 in $\gamma^{\alpha \beta}$ and denominator $\left(\operatorname{det} \gamma^{\alpha \beta}\right)^{2}$. If the condition (4.1) is satisfied, $R^{\mu \nu}$ reduces to $R_{h}^{\mu v}$ and the vacuum Einstein equations are equivalent to:

$$
\gamma^{\alpha \beta} D_{\alpha} D_{\beta} \gamma^{\mu v}=B^{\mu v}\left(\gamma^{\alpha \beta}, D_{\varrho} \gamma^{\alpha \beta}\right)
$$

The differential system (4.6) is a weakly coupled quasilinear hyperbolic system of the second order. This system is called the reduced Einstein equations.

In this section we shall demonstrate the existence of global systems of harmonic coordinates on spacetimes which are globally hyperbolic and satisfy the largeness requirement of the boost problem. We shall also show, following Y. ChoquetBruhat [2], the preservation of the harmonic coordinate condition by solutions of the reduced Einstein equations whose Cauchy data satisfy the constraints. We shall use the theorems derived in [22], on weakly coupled linear hyperbolic systems of the second order in such spacetimes of $n$ dimensions. 
Let $\Omega_{\theta}$ be the open subset of $\mathbb{R}^{n}$ defined by (2.2). The spacetime $\left(\Omega_{\theta}, \eta\right)$, where $\eta \equiv \operatorname{diag}(-1,+1, \ldots 2,+1)$ is the Minkowski metric on $\mathbb{R}^{n}$, is globally hyperbolic and satisfies the largeness requirement of the boost problem. For each $x \in \Sigma$ :

$$
\tilde{\chi}^{ \pm}(x)=\theta\left[1+\frac{(\tilde{d}(x))^{2}}{1-\theta^{2}}\right]^{1 / 2} \text {. }
$$

In fact, $\tilde{\chi}^{ \pm}(x)$ is the length of the timelike geodesic orthogonal to the boundary $\Sigma_{\theta}$ of $\Omega_{\theta}$ and $\tilde{d}(x)=|\bar{x}|$. The function $\tau$ defined by (2.3) is a time function on $\left(\Omega_{\theta}, \eta\right)$. Each $\Sigma_{\tau}$ is a complete Cauchy surface of this spacetime and we have $\Sigma_{0}=\Sigma$. Using the foliation $\left\{\Sigma_{\tau} \mid \tau \in I_{\theta}\right\}$ we shall define a class of Lorentz metrics $\gamma$ on $\Omega_{\theta}$ such that $\left(\Omega_{\theta}, \gamma\right)$ is also a globally hyperbolic spacetime satisfying the largeness requirement of the boost problem. Let $\tilde{n}_{\mu}$ denote the gradient of $\tau$ normalized with respect to $\eta$. We have:

$$
\tilde{n}_{\mu}=-\tilde{N} D_{\mu} \tau
$$

where $\tilde{N}$ is the lapse function of the foliation relative to $\eta$ :

$$
\tilde{N}^{-2}=-\eta^{\mu v} D_{\mu} \tau D_{\nu} \tau, \tilde{N}>0 .
$$

Definition 4.1. A $C^{0}$ 2-covariant symmetric tensorfield $\gamma^{\mu v}$ defined on $\Omega_{\theta}$ is a regularly hyperbolic metric if there exist positive real numbers $a, b$ and $C$ such that in $\Omega_{\theta}$ :

(1) $-\gamma^{\mu v} \tilde{n}_{\mu} \tilde{n}_{\nu} \geqq a$

(2) for all covectors $\zeta_{\mu}$ such that $\gamma^{\mu v} \zeta_{\mu} \tilde{n}_{v}=0$, we have:

$$
\gamma^{\mu v} \zeta_{\mu} \zeta_{\nu} \geqq b|\zeta|^{2}
$$

(3) $|\gamma| \leqq C$.

In the above definition, requirement (1) implies that the vectorfield $\gamma^{\mu v} \tilde{n}_{v}$ is timelike. Requirement (2) implies that the metric induced on the tangent space to $\Sigma_{\tau}$ at each point, is positive definite for every $\tau \in I_{\theta}$. Finally, requirement (3) ensures that these two statements are true in a uniform sense. Let us set:

$$
h=\max \left\{\frac{1}{a}, \frac{1}{b}, C\right\} .
$$

We shall call $h$ the coefficient of regular hyperbolicity of $\gamma$.

The usefulness of Definition (4.1) can be seen in:

Proposition 4.1. If $\gamma$ is a regularly hyperbolic metric on $\Omega_{\theta}$, then $\left(\Omega_{\theta}, \gamma\right)$ is a globally hyperbolic spacetime satisfying the largeness requirement of the boost problem.

This proposition was proven in [22]. The proof involves finding a lower bound to the length of the integral curve of the unit normal vectorfield $n^{\mu}$, relative to $\gamma^{\mu \nu}$, to the foliation $\left\{\Sigma_{\tau} \mid \tau \in I_{\theta}\right\}$, through each point in $\Sigma$. We have also:

Proposition 4.2. The set of regularly hyperbolic metrics on $\Omega_{\theta}$ is open in the Banach space $C_{b}^{0}\left(\Omega_{\theta}\right)$ of continuous and bounded 2-contravariant symmetric tensorfields on $\Omega_{\theta}$. 
The Minkowski metric $\eta$ is obviously regularly hyperbolic on $\Omega_{\theta}$. The numbers $a, b$, and $C$ for $\eta$ may be chosen to be:

$$
a=1, \quad b=1-\theta^{2}, \quad C=(n)^{1 / 2} .
$$

By Proposition 4.2 there exists a positive real number $\varepsilon$ such that if $\gamma$ is a $C^{0}$ 2-contravariant symmetric tensorfield on $\Omega_{\theta}$ and $\left.\mid \gamma-\eta\right) \leqq \varepsilon$, then $\gamma$ is a regularly hyperbolic metric on $\Omega_{\theta}$.

Let now $L$ be a linear differential operator of the second order in $\Omega_{\theta}$ :

$$
L u=\sum_{k=0}^{2} a_{k} \cdot D^{k} u
$$

where $u$ and $L u$ are $\mathbb{R}^{n}$ valued functions on $\Omega_{\theta}$. We introduce the following hypotheses:

Hypothesis I (weak coupling and hyperbolicity).

We have:

$$
a_{2}=\gamma I
$$

that is, in components,

$$
\left(a_{2}\right)_{J}^{\mu \nu I}=\gamma^{\mu \nu} \delta_{J}^{I}
$$

where $\gamma$ is a regularly hyperbolic metric on $\Omega_{\theta}$.

Hypothesis II (regularity). There exist nonnegative integers $s_{k}$ and real numbers $\delta_{k}$, $0 \leqq k \leqq 2$ such that:

$$
s_{k}>\frac{n}{2}+k-1, \delta_{k}>2-k-\frac{n}{2}: 0 \leqq k \leqq 2
$$

and

(1) $a_{k} \in H_{s_{k}, \delta_{k}}\left(\Omega_{\theta}\right): k=0,1$

(2) $\gamma-\eta \in H_{s_{2}, \delta_{2}}\left(\Omega_{\theta}\right)$.

We shall write:

$$
m=\sum_{k=0}^{1}\left\|a_{k}\right\|_{H_{s_{k}, \delta_{k}}\left(\Omega_{\theta}\right)}+\|\gamma-\eta\|_{H_{s_{2}, \delta_{2}}\left(\Omega_{\theta}\right)}
$$

and (see Lemma 2.4 (restriction)) :

$$
\mu=\sum_{k=0}^{1}\left\|a_{k}\right\|_{H_{s_{k}-1, \delta_{k}+\frac{1}{2}\left(\Sigma, \Omega_{\theta}\right)}}+\|\gamma-\eta\|_{s_{2}-1, \hat{o}_{2}+\frac{1}{2}\left(\Sigma, \Omega_{\theta}\right)} .
$$

Let us also denote:

$$
s^{\prime}=\min _{0 \leqq k \leqq 2}\left\{s_{k}\right\}+1 .
$$

Hypothesis II implies that $L$ is a continuous map:

$$
H_{s+1, \delta}\left(\Omega_{\theta}\right) \rightarrow H_{s-1, \delta+2}\left(\Omega_{\theta}\right)
$$

for

$$
1 \leqq s \leqq s^{\prime}, \quad \delta \in \mathbb{R}
$$


We shall give below an extended version of the theorems on weakly coupled linear hyperbolic systems of the second order which were proven in [22]. The proof of these theorems is based on the use of energy estimates with weights. These estimates are derived using the foliation $\left\{\Sigma_{\tau} \mid \tau \in I_{\theta}\right\}$. It is important in this derivation the fact that the ratio $N / \bar{\sigma}$, of the lapse function of this foliation relative to $\gamma$ :

$$
N^{-2}=-\gamma^{\mu \nu} D_{\mu} \tau D \tau, N>0
$$

to the weight function $\bar{\sigma}$, is, by virtue of Definition 4.1 , bounded above and below by positive constants. The following is an outline of the proof of the theorems. Given a linear differential operator $L$, of second order, satisfying hypotheses I and II, and given a function $u \in H_{s+1, \delta}\left(\Omega_{\theta}\right), 1 \leqq s \leqq s^{\prime}, \delta \in \mathbb{R}$, one first derives a weighted energy estimate of the form:

$$
\|u\|_{H_{s, \delta}\left(\Omega_{\delta}\right)} \leqq c_{1} \theta^{1 / 2}\left\{\|u\|_{H_{s, \delta+\frac{1}{2}\left(\Sigma, \Omega_{\theta}\right)}}+\theta^{1 / 2}\|\beta\|_{H_{s-1}, \delta+2\left(\Omega_{\theta}\right)}\right\}
$$

where:

$$
\beta=L u
$$

and $c_{1}$ is a continuous monotonically increasing function of $\left(\theta, h, \theta^{1 / 2} m\right)$. One then uses the restriction to the hypersurface $\Sigma$ of the system $L u=\beta$ and its differential consequences to bound:

$$
\begin{aligned}
& \|u\|_{H_{s, \delta+\frac{1}{2}\left(\Sigma, \Omega_{\theta}\right)} \leqq c_{2}\left\{\|\phi\|_{H_{S, \delta+\frac{1}{2}(\Sigma)}}\right.} \\
& \quad+\|\psi\|_{H_{S-1, \delta+\frac{3}{2}(\Sigma)}}+\|\beta\|_{\left.H_{S-2, \delta+\frac{5}{2}\left(\Sigma, \Omega_{\theta}\right)}\right\}}
\end{aligned}
$$

where:

$$
\phi=u_{\mid \Sigma}, \quad \psi=D_{0} u_{\mid \Sigma}
$$

and $c_{1}$ is a continuous monotonically increasing function of $(h, \mu)$. Combining inequalities (4.11) and (4.12) one obtains:

$$
\begin{aligned}
& \|u\|_{H_{s, \delta}\left(\Omega_{\theta}\right)} \leqq c_{1} \theta^{1 / 2}\left\{c _ { 2 } \left[\|\phi\|_{H_{s, \delta+\frac{1}{2}(\Sigma)}}+\|\psi\|_{H_{s-1, \delta+\frac{3}{2}(\Sigma)}}\right.\right. \\
& \quad+\|\beta\|_{\left.\left.H_{s-2, \delta+\frac{5}{2}\left(\Sigma, \Omega_{\theta}\right)}\right]+\theta^{1 / 2}\|\beta\|_{H_{s-1, \delta+2}\left(\Omega_{\theta}\right)}\right\} .}
\end{aligned}
$$

The uniqueness theorem follows directly from this inequality. To prove the existence theorem one takes a sequence of operators $\left\{L_{n}\right\}$ satisfying hypothesis I, such that $\left(a_{k}\right)_{n}: k=0,1$ and $\gamma_{n}-\eta$ are restrictions to $\Omega_{\theta}$ of functions in $C_{0}^{\infty}\left(\mathbb{R}^{n}\right)$ and:

$$
\begin{gathered}
\left(a_{k}\right)_{n} \rightarrow a_{k} \in H_{s_{k}, \delta_{k}}\left(\Omega_{\theta}\right): k=0,1, \\
\gamma_{n}-\eta \rightarrow \gamma-\eta \in H_{s_{2}, \delta_{2}}\left(\Omega_{\theta}\right) .
\end{gathered}
$$

One also takes a sequence of inhomogeneous terms $\left\{\beta_{n}\right\}$ such that $\beta_{n}$ is the restriction to $\Omega_{\theta}$ of a function in $C_{0}^{\infty}\left(\mathbb{R}^{n}\right)$ and:

$$
\beta_{n} \rightarrow \beta \in H_{s-1, \delta+2}\left(\Omega_{\theta}\right),
$$

as well as a sequence of data $\left(\phi_{n}, \psi_{n}\right)$ in $C_{0}^{\infty}(\Sigma)$ such that:

$$
\left(\phi_{n}, \psi_{n}\right) \rightarrow(\phi, \psi) \in H_{s, \delta+\frac{1}{2}}(\Sigma) \times H_{s-1, \delta+\frac{3}{2}}(\Sigma) .
$$


Since for each $n\left(\Omega_{\theta}, \gamma_{n}\right)$ is a globally hyperbolic spacetime, it results from the Leray theory [4] that there exists a unique solution $u_{n} \in C^{\infty}\left(\Omega_{\theta}\right)$ to:

$$
L_{n} u_{n}=\beta_{n},
$$

taking on $\Sigma$ the Cauchy data $\left(\phi_{n}, \psi_{n}\right)$ :

$$
u_{n \mid \Sigma}=\phi_{n}, \quad D_{0} u_{n \mid \Sigma}=\psi_{n} .
$$

From the support properties of the solution it follows in particular that $u_{n} \in H_{s+1, \delta}\left(\Omega_{\theta}\right)$ and therefore inequality (4.13) applies. The proof then relies on the weak compactness of the closed balls of the Hilbert space $H_{s, \delta}\left(\Omega_{\theta}\right)$. Details are found in [22].

Theorem 4.1 (existence and estimate for the linear system). Let $L$ be a differential operator of second order in $\Omega_{\theta}$ satisfying hypotheses I and II. Let (inhomogeneous term) $\beta \in H_{s-1, \delta+2}\left(\Omega_{\theta}\right)$ and (Cauchy data) $\phi \in H_{s, \delta+\frac{1}{2}}(\Sigma), \psi \in H_{s-1, \delta+\frac{3}{2}}(\Sigma)$ where $2 \leqq s \leqq s^{\prime}, \delta \in \mathbb{R}$. Then there exists at least one solution $u \in H_{s, \delta}\left(\Omega_{\theta}\right)$ to:

$$
L u=\beta,
$$

taking on $\Sigma$ the Cauchy data $(\phi, \psi)$ :

$$
u_{\mid \Sigma}=\phi, \quad D_{0} u_{\mid \Sigma}=\psi
$$

and satisfying the estimate:

$$
\begin{aligned}
& \|u\|_{H_{s, \delta}\left(\Omega_{\theta}\right)} \leqq c_{1} \theta^{1 / 2}\left\{c _ { 2 } \left[\|\phi\|_{H_{s, \delta+\frac{1}{2}(\Sigma)}}+\|\psi\|_{H_{s-1, \delta+\frac{3}{2}(\Sigma)}}\right.\right. \\
& \quad+\|\beta\|_{\left.\left.H_{s-2, \delta+\frac{5}{2}\left(\Sigma, \Omega_{\theta}\right)}\right]+\theta^{1 / 2}\|\beta\|_{H_{s-1, \delta+2}\left(\Omega_{\theta}\right)}\right\},}
\end{aligned}
$$

where $c_{1}$ and $c_{2}$ are continuous monotonically increasing functions of $\left(\theta, h, \theta^{1 / 2} m\right)$ and $(h, \mu)$ respectively.

Theorem 4.2. (uniqueness for the linear system). Let $L$ be a differential operator in $\Omega_{\theta}$ satisfying hypotheses I and II. Then there is at most one solution $u \in H_{2, \delta}\left(\Omega_{\theta}\right)$, $\delta \in \mathbb{R}$, to :

$$
L u=\beta ; \quad u_{\mid \Sigma}=\phi, \quad D_{0} u_{\mid \Sigma}=\psi,
$$

where $\beta$ is a given function on $\Omega_{\theta}$ and $\phi, \psi$ are given functions on $\Sigma$.

We shall now use the above theorems to prove the following theorem on harmonic coordinates:

Theorem 4.3 (harmonic coordinates). Let $s>n / 2+1, \delta>-n / 2$ and let $\gamma$ be $a$ regularly hyperbolic metric on $\Omega_{\theta_{0}}$ such that $\gamma-\eta \in H_{s+1, \delta}\left(\Omega_{\theta_{0}}\right)$. Then there exists a $\left.\theta \in] 0, \theta_{0}\right]$ and a unique coordinate transformation $\xi: \Omega_{\theta} \rightarrow \Omega^{\prime}$, by $x \rightarrow x^{\prime}=\xi(x)$, such that $x^{\prime}$ is a harmonic coordinate system on $\left(\Omega^{\prime}, \gamma^{\prime}\right), \gamma^{\prime} \equiv \xi_{*} \gamma$ is Gaussian on $\Sigma$, $\xi$ is the identity on $\Sigma$ and $\xi-\mathrm{id} \in H_{s+1, \delta-1}\left(\Omega_{\theta}\right)$. If $\|\gamma-\eta\|_{H_{s+1, \delta}\left(\Omega_{\theta_{v}}\right)}$ is sufficiently small, $\theta$ can be chosen equal to $\theta_{0}$.

Proof. The requirement that $x^{\prime}$ be harmonic relative to $\gamma^{\prime}$ forms an $n$-tuplet of scalar equations :

$$
\square_{\gamma^{\prime}} x^{\prime \mu}=0
$$


Consequently, it is equivalent to:

$$
\square_{\gamma} \xi^{\mu}=0
$$

Let us set:

$$
\xi^{\mu}=x^{\mu}+f^{\mu}
$$

Equations (4.14) then become:

$$
\square_{\gamma} f^{\mu} \equiv\left(\gamma^{\alpha \beta} D_{\alpha} D_{\beta}-\Gamma^{\alpha} D_{\alpha}\right) f^{\mu}=\Gamma^{\mu},
$$

where:

$$
\Gamma^{\mu}=-\square_{\gamma} x^{\mu}=\gamma^{\alpha \beta} \Gamma_{\alpha \beta}^{\mu} .
$$

The operator $\square_{\gamma}$ in (4.15) is a linear differential operator of second order in $\Omega_{\theta_{0}}$. By the assumptions of the theorem, this operator satisfies hypotheses I and II. Since we require $\xi$ to be the identity on $\Sigma$, we have:

$$
f_{\mid \Sigma}=0 \text {. }
$$

Then the metric $\gamma^{\prime}$ is Gaussian on $\Sigma$, that is :

$$
\gamma_{\mid \Sigma}^{\prime 00}=-1, \quad \gamma_{\mid \Sigma}^{\prime 0 i}=0
$$

if and only if:

where:

$$
D_{0} f_{\mid \Sigma}=1
$$

$$
l^{0}=\frac{1}{\left(-\gamma_{\mid \Sigma}^{00}\right)^{1 / 2}}-1, \quad l^{i}=-\frac{\gamma_{\mid \Sigma}^{0 i}}{\gamma_{\mid \Sigma}^{00}} .
$$

By the restriction lemma (Lemma 2.4) and the multiplication theorem we have: $l \in H_{s, \delta+\frac{1}{2}}(\Sigma)$. Finally, the inhomogeneous term $\Gamma^{\mu}$ in (4.15) belongs to $H_{s, \delta+1}\left(\Omega_{\theta_{0}}\right)$. It follows that we may apply Theorems 4.1 and 4.2 to the problem posed by (4.15), (4.16) and (4.17). We thus obtain a unique solution $f \in H_{s+1, \delta-1}\left(\Omega_{\theta_{0}}\right)$. Furthermore (using again the restriction lemma), this solution satisfies a bound of the form:

$$
\|f\|_{H_{s+1, \delta-1}\left(\Omega_{\theta_{0}}\right)} \leqq r B
$$

where :

$$
r=\|\gamma-\eta\|_{H_{s+1, \delta}\left(\Omega_{\theta_{0}}\right)}
$$

and $B$ depends continuously and in a monotonically increasing fashion on $r$. The imbedding theorem then implies that $D^{2} f \in C_{1}^{0}\left(\Omega_{\theta_{0}}\right)$ and:

$$
\left\|D^{2} f\right\|_{C_{1}^{0}\left(\Omega_{\theta_{0}}\right)} \leqq r B^{\prime},
$$

where $B^{\prime}$ depends continuously and in a monotonically increasing fashion on $r$. Let us denote:

$$
J=D \xi=I+D f .
$$

Taking into account the fact that $\partial / \partial \tau=\bar{\sigma} \partial / \partial x^{0}$, we have for every $\tau \in I_{\theta_{0}}$ :

$$
|J(\tau)-J(0)| \leqq|\tau|\|D J\|_{C_{1}^{0}\left(\Omega_{\theta_{0}}\right)}
$$


and therefore

$$
|J(\tau)-J(0)| \leqq|\tau| r B^{\prime}
$$

in view of inequality (4.19). $J(0)$ is the following $n \times n$-matrix valued function on $\mathbb{R}^{n-1}$.

$$
J(0)=\left(\begin{array}{cc}
1+l^{0} & 0 \\
l^{i} & \delta_{j}^{i}
\end{array}\right) .
$$

We have:

$$
\operatorname{det} J(0)=1+l^{0}=\left(-\gamma_{\mid \Sigma}^{00}\right)^{-1 / 2} \geqq 1 / h,
$$

using Definition 4.1. There exists a positive real number $\varepsilon$ such that $|J(\tau)-J(0)| \leqq \varepsilon$ on $\mathbb{R}^{n-1}$ implies that $\operatorname{det} J(\tau)$ is also bounded below by a positive constant. Hence choosing $\theta>0, \theta \leqq \theta_{0}$, such that:

$$
\theta r B^{\prime}<\varepsilon,
$$

we have by virtue of inequality (4.20):

$$
\operatorname{det} J \geqq c>0
$$

in $\Omega_{\theta}$. It then follows from the global implicit function theorem (see [27]) that the restriction of $\xi$ to $\Omega_{\theta}$ (which we continue to denote by $\xi$ ) is a diffeomorphism of $\Omega_{\theta}$ into its image $\Omega^{\prime} \equiv \xi\left(\Omega_{\theta}\right)$. Furthermore, it is evident from (4.21) that if $r$ is sufficiently small we may choose $\theta=\theta_{0}$.

Applying the multiplication theorem, Lemma 2.3 and the composition theorem to the formula for the pushout $\gamma^{\prime}=\xi_{*} \gamma$ :

$$
\gamma^{\prime \mu \nu}=\left(D_{\alpha} \xi^{\mu} D_{\beta} \xi^{v} \gamma^{\alpha \beta}\right) \circ \xi^{-1}
$$

we conclude that: $\gamma^{\prime}-\eta \in H_{s, \delta}\left(\Omega^{\prime}\right)$. The fact that $\xi-\mathrm{id} \in C_{\beta-1}^{0}\left(\Omega_{\theta}\right)$ for some $\beta>0$ (imbedding theorem) implies that there exists a $\theta^{\prime}>0$ such that $\Omega_{\theta^{\prime}} \subset \Omega^{\prime}$.

Let us now return to the reduced Einstein equations (4.6). Cauchy data for these equations consists of giving:

$$
\gamma_{\mid \Sigma}=\phi, \quad D_{0} \gamma_{\mid \Sigma}=\psi \text {. }
$$

From given initial data $(g, k)$ we construct Cauchy data $(\phi, \psi)$ satisfying $\Gamma_{\mid \Sigma}^{\mu}=0$. There is arbitrariness in this construction which corresponds to the freedom in choosing a harmonic coordinate system. A canonical prescription, in the framework of Theorem 4.3, is to require that the coordinate system is also Gaussian on $\Sigma$, that is:

$$
\begin{gathered}
\phi^{00}=-1 \\
\phi^{0 i}=0 .
\end{gathered}
$$

With this choice we have:

$$
\phi^{i j}=g^{i j} .
$$


The condition $\Gamma_{\mid \Sigma}^{\mu}=0$ then defines:

$$
\begin{gathered}
\psi^{00}=-4 \operatorname{tr}_{g} k \\
\psi^{0 i}=-\Delta_{g} x^{i}
\end{gathered}
$$

and we have also:

$$
\psi^{i j}=2 g^{i m} g^{j n} k_{m n} .
$$

In the language of ADM [28] we are free to choose the lapse $N$ and the shift $\zeta^{i}$ on $\Sigma$. We make the choice $N_{\mid \Sigma}=1, \zeta_{\mid \Sigma}^{i}=0$. The condition $\Gamma_{\mid \Sigma}^{\mu}=0$ then defines:

$$
D_{0} N_{\mid \Sigma}=-2 \operatorname{tr}_{g} k, \quad D_{0} \zeta_{\mid \Sigma}^{i}=-\Delta_{g} x^{i} .
$$

If $(g-e, k) \in H_{s, \delta+\frac{1}{2}}(\Sigma) \times H_{s-1, \delta+\frac{3}{2}}(\Sigma)$, then by the multiplication theorem we have $(\phi-\eta, \psi) \in H_{s, \delta+\frac{1}{2}}(\Sigma) \times H_{s-1, \delta+\frac{3}{2}}(\Sigma)$ provided that $s \geqq 2, \delta>-2$.

Let us now be given a regularly hyperbolic metric $\gamma$ in $\Omega_{\theta}$ which is a solution of the reduced Einstein equations $R_{h}^{\mu \nu}=0$, such that $\gamma-\eta \in H_{s, \delta}\left(\Omega_{\theta}\right)$ with $s \geqq 4, \delta>-2$. Let the Cauchy data of $\gamma$ satisfy $\Gamma_{\mid \Sigma}^{\mu}=0$ as well as the initial value constraints $G_{\mid \Sigma}^{\mu 0}=0$. It then follows directly from expression (4.2) that we have also $D_{0} \Gamma_{\mid \Sigma}^{\mu}=0$. Furthermore since by the multiplication theorem the Einstein tensor $G^{\mu \nu}$ of $\gamma$ belongs to $H_{s-2, \delta+2}\left(\Omega_{\theta}\right)$, we may use the Bianchi identities $G_{; v}^{\mu v}=0$ to derive from expression (4.2), as in Choquet-Bruhat [2], the following differential condition on $\Gamma^{\mu}$ :

$$
\gamma^{\alpha \beta} D_{\alpha} D_{\beta} \Gamma^{\mu}+B_{\beta}^{\mu \alpha}\left(\gamma^{\kappa \lambda}, D_{\varrho} \gamma^{\kappa \lambda}\right) D_{\alpha} \Gamma^{\beta}=0
$$

where:

$$
B_{\beta}^{\mu \alpha}=Q_{\beta, \kappa \lambda}^{\mu \alpha, \varrho} D_{\varrho} \gamma^{\kappa \lambda}
$$

and $Q$ is a rational function of $\gamma^{\kappa \lambda}$, with numerator a homogeneous polynomial of degree 4 in $\gamma^{\kappa \lambda}$ and denominator $\operatorname{det} \gamma^{\kappa \lambda}$. By the multiplication theorem, $B$ belongs to $H_{s-1, \delta+1}\left(\Omega_{\theta}\right)$. Thus equations (4.25) form a homogeneous differential system satisfying hypotheses I and II. Hence we may apply Theorem 4.2 to conclude $\Gamma^{\mu}=0$ on $\Omega_{\theta}$, which yields the following lemma :

Lemma 4.1. Let $s \geqq 4, \delta>-2$ and let $\gamma$ be a regularly hyperbolic metric in $\Omega_{\theta}$ such that $\gamma-\eta \in H_{s, \delta}\left(\Omega_{\theta}\right)$. If $\gamma$ satisfies the reduced Einstein equations in $\Omega_{\theta}$ and its Cauchy data $(\phi, \psi)$ satisfy the initial value constraints as well as $\Gamma_{\mid \Sigma}^{\mu}=0$, then $\gamma$ satisfies the harmonicity condition $\Gamma^{\mu}=0$ everywhere in $\Omega_{\theta}$. Thus $\gamma$ is a solution of the full Einstein equations in $\Omega_{\theta}$.

\section{The Reduced Einstein Equations}

In this section we shall demonstrate the existence and uniqueness of regularly hyperbolic solutions $\gamma$ to the reduced Einstein equations in a domain $\Omega_{\theta}$, taking on $\Sigma$ given asymptotically flat Cauchy data. Our method is based on the study of the boost problem for weakly coupled quasilinear hyperbolic systems of the second order in [22]. These differential systems have the form:

$$
\begin{gathered}
\gamma^{\mu v}(u, D u) D_{\mu} D_{v} u^{I}=\beta^{I}(u, D u) \\
\mu, v=0, \ldots, n-1: \quad I=1, \ldots, N,
\end{gathered}
$$


where $\gamma(u, v)$ and $\beta(u, v)$ are functions of $(u, v)$ defined in an open subset $Y$ of $\mathbb{R}^{N} \times \mathbb{R}^{n N}$ and $\gamma$ is a Lorentz matrix in $Y$. We assume that $\gamma$ and $\beta$ are $C^{\infty}$ functions of $(u, v)$ in $Y$, that $(0,0) \in Y$ and that $\gamma(0,0)=\eta$. In the study of the boost problem for the above systems the notion of degree of the function $\beta$ plays an essential role. This notion is defined by the following definitions:

Definition 5.1. Let $\beta$ be a $C^{\infty}$ function of $(u, v)$ in $Y$. We say that $\beta$ is of type $(p, q)$ if:

$$
\partial^{i} \beta / \partial u^{i}=0 \text { on the plane } u=0, \text { for all } 0 \leqq i \leqq p-1
$$

and

$$
\partial^{j} \beta / \partial v^{j}=0 \text { on the plane } v=0 \text {, for all } 0 \leqq j \leqq q-1 .
$$

Thus $\beta$ is of type $(p, q)$ if there exists a $C^{\infty}$ function $\bar{\beta}$ of $(u, v)$ in $Y$ such that:

$$
\beta(u, v)=u^{p} v^{q} \cdot \bar{\beta}(u, v) .
$$

Definition 5.2. If $\beta(u, v)$ is of type $(p, q)$ with $p+q \geqq 2$, we say that $\beta$ is of degree $\varepsilon$, where:

$$
\varepsilon=\max \left\{0, \frac{2-q}{p+q-1}\right\} .
$$

Definition 5.3. Let $\beta(u, v)$ be a finite sum:

$$
\beta=\sum_{i=1}^{M} \beta_{i},
$$

where, for each $1 \leqq i \leqq M, \beta_{i}$ is of type $\left(p_{i}, q_{i}\right)$ with $p_{i}+q_{i} \geqq 2$. Then we say that $\beta$ is of degree $\varepsilon$, where:

$$
\varepsilon=\max _{1 \leqq i \leqq M}\left\{\varepsilon_{i}\right\}
$$

$\varepsilon_{i}$ being the degree of $\beta_{i}$.

From the above definitions it follows that $\varepsilon$ is a rational number in the closed interval $[0,2]$.

We shall give below an extended version of the theorem, proven in [22], on the boost problem for quasilinear hyperbolic systems of the form (5.1). The following is an outline of the method of proof. Given a function $u^{\prime}$ defined on some $\Omega_{\theta}$ and satisfying:

(a) $\left\{\left(u^{\prime}(x), D u^{\prime}(x)\right) \mid x \in \Omega_{\theta}\right\} \subset Y$, we associate with the quasilinear system (5.1) the linear system obtained by replacing in all terms except the second derivatives $u$ by $u^{\prime}$ :

$$
\gamma\left(u^{\prime}, D u^{\prime}\right) \cdot D^{2} u=\beta\left(u^{\prime}, D u^{\prime}\right)
$$

This is a weakly coupled linear hyperbolic system of the second order in $\Omega_{\theta}$. If the given function $u^{\prime}$ is such that:

(b) $\gamma\left(u^{\prime}, D u^{\prime}\right)$ is a regularly hyperbolic metric on $\Omega_{\theta}$, then the differential operator $\gamma\left(u^{\prime}, D u^{\prime}\right) \cdot D^{2}$ satisfies hypothesis I of Sect. 4. If $u \in H_{s, \delta}\left(\Omega_{\theta}\right)$ with $s>n / 2+1$, $\delta>-n / 2$, the multiplication theorem implies: $\gamma\left(u^{\prime}, D u^{\prime}\right)-\eta \in H_{s-1, \delta}\left(\Omega_{\theta}\right)$, which shows that if $s>n / 2+2$ also the operator $\gamma\left(u^{\prime}, D u^{\prime}\right) \cdot D^{2}$ satisfies hypothesis II of 
Sect. 4 as well (we have $a_{k}=0$ for $k=0,1$ ). To apply Theorem 4.1 to the linear system (5.2) we need to have: $\beta\left(u^{\prime}, D u^{\prime}\right) \in H_{s-1, \delta+2}\left(\Omega_{\theta}\right)$. This is the point where the notion of degree comes in; one shows, using the multiplication theorem, that if $\beta$ is of degree $\varepsilon$ then $u^{\prime} \in H_{s . \delta}\left(\Omega_{\theta}\right)$ with $s>n / 2+1, \delta>\varepsilon-n / 2$ implies $\beta\left(u^{\prime}, D u^{\prime}\right) \in H_{s-1, \delta+2}\left(\Omega_{\theta}\right)$. It follows that for $s<n / 2+2, \delta>\varepsilon-n / 2$ and Cauchy data : $(\phi, \psi) \in \mathrm{H}_{s, \delta+\frac{1}{2}}(\Sigma) \times \mathrm{H}_{s-1, \delta+\frac{3}{2}}(\Sigma)$,

$$
u_{\mid \Sigma}=\phi, \quad D_{0} u_{\mid \Sigma}=\psi,
$$

we may apply Theorems 4.1 and 4.2 to the linear system (5.2) to conclude that there exists a unique solution $u \in H_{s, \delta}\left(\Omega_{\theta}\right)$ to (5.2) taking on $\Sigma$ the given Cauchy data. Hence we have a nonlinear map $T: u^{\prime} \rightarrow u$ from the subset of $H_{s, \delta}\left(\Omega_{\theta}\right)$ defined by conditions (a) and (b) into $H_{s, \delta}\left(\Omega_{\theta}\right)$.

To define an iteration using $T$, we have to show that also $u$ satisfies conditions (a) and (b) above. For this purpose it is necessary that the Cauchy data $(\phi, \psi)$ satisfy :

and

(I) $\{(\phi(x), \psi(x)) \mid x \in \Sigma\} \subset Y$

(II) $\Sigma$ is spacelike for $\gamma(\phi, \psi)=\gamma_{\mid \Sigma}$, in the sense of the following definition

Definition 5.4. The hypersurface $\Sigma$ is spacelike for $\gamma_{\mid \Sigma}$ if on $\Sigma$ :

(1) $\gamma^{00}<0$

(2) for every covector $\xi_{\mu}$ such that $\gamma^{0 \mu} \xi_{\mu}=0$ we have:

$$
\gamma^{\mu v} \xi_{\mu} \xi_{v}>0
$$

Given data $(\phi, \psi) \in H_{s, \delta+\frac{1}{2}}(\Sigma) \times H_{s-1, \delta+\frac{3}{2}}(\Sigma)$ with $s>n / 2+1, \delta>-n / 2$, the imbedding theorem implies that $(\phi, \psi) \in C^{1}(\Sigma) \times C^{0}(\Sigma)$ and $(\phi, \psi) \rightarrow(0,0)$ for $|x| \rightarrow \infty$. Hence, since $\gamma$ is a $C^{\infty}$ function of $(u, v)$ in $Y$ and $(0,0) \in Y$, the above requirements (I) and (II) imply:

(I') $\{(\phi(x), \psi(x)) \mid x \in \Sigma\} \subset \bar{Y}_{0}$,

where $\bar{Y}_{0}$ is a compact subset of $Y$, and

$\left(\mathrm{II}^{\prime}\right) \gamma(\phi, \psi)$ is a regularly hyperbolic metric on $\Sigma$, that is $\gamma(\phi, \psi)$ satisfies on $\Sigma$ the requirements of Definition (4.1). Now let $u \in H_{s, \delta}\left(\Omega_{\theta}\right)$ with $s>n / 2+2, \delta>-\mathrm{n} / 2$, which implies $\gamma(u, D u)-\eta \in H_{s-1, \delta}\left(\Omega_{\theta}\right)$. By the imbedding theorem the partial derivatives of $u, D u$ and $\gamma$ with respect to $\tau$ are all continuous and bounded in $\Omega_{\theta}$. Using this fact one can show from $\left(\mathrm{I}^{\prime}\right)$ and $\left(\mathrm{II}^{\prime}\right)$, in view of the open ness of $Y$ and the definition of regular hyperbolicity (Proposition 4.2), that if $\theta$ is sufficiently small $u$ satisfies conditions (a) and (b):

(a) $\left\{(u(x), D u(x)) \mid x \in \Omega_{\theta}\right\} \subset Y$, and

(b) $\gamma(u, D u)$ is a regularly hyperbolic metric in $\Omega_{\theta}$. These conditions also hold for any given $\theta, 0<\theta<1$, if $\|u\|_{H_{s, \delta}\left(\Omega_{\theta}\right)}$ is sufficiently small. We conclude that the nonlinear map $T$ may be used to define an iteration.

We now consider the closed subset $S_{r}$ of $H_{s, \delta}\left(\Omega_{\theta}\right), s>n / 2+2, \delta>\varepsilon-n / 2$, consisting of those functions $u$ in the closed ball of radius $r$ which take on $\Sigma$ the Cauchy data $(\phi, \psi)$ and which are such that $D_{0}^{i} u_{\mid \Sigma}$ for $2 \leqq i \leqq s-1$ is calculated from 
$(\phi, \psi)$ using the quasilinear system (5.1) on the hypersurface $\Sigma$. For $u \in S_{r}$ we can bound the quantities:

$$
\mu=\|\gamma(u, D u)-\eta\|_{H_{s-2, \delta+\frac{1}{2}\left(\Sigma, \Omega_{\theta}\right)}}
$$

and

$$
\|\beta(u, D u)\|_{H_{s-2, \delta+\frac{5}{2}\left(\Sigma, \Omega_{\theta}\right)}},
$$

which enter the estimate in Theorem 4.1, in terms of the size of the data:

$$
d=\|\phi\|_{H_{s, \delta+\frac{1}{2}(\Sigma)}}+\|\psi\|_{H_{s-1, \delta+\frac{3}{2}(\Sigma)}}
$$

and $\bar{Y}_{0}$. Application of the estimate in Theorem 4.1 shows that if either $\theta$ or $d$ is sufficiently small the map $T$ sends $S_{r}$ into itself for some $r$. Thus if we start with any $u_{0} \in S_{r}$ the sequence of iterates $\left\{u_{n} \mid n \in N\right\}$, where $u_{n+1}=T\left(u_{n}\right)$, is contained in $S_{r}$. One then shows, using again the estimate in Theorem 4.1, that $T$ is a contracting map with respect to the $H_{1, \delta}$ norm (in fact with respect to the $H_{s-1, \delta}$ norm) in $S_{r}$. The proof then relies on the contraction mapping principle and the weak compactness of the closed ball of radius $r$ in the Hilbert space $H_{s, \delta}\left(\Omega_{\theta}\right)$. Details are found in [22].

Theorem 5.1. (existence and uniqueness for the quasilinear system). Let $\gamma(u, v)$ and $\beta(u, v)$ be $C^{\infty}$ functions of $(u, v)$ in an open set $Y \subset \mathbb{R}^{N} \times \mathbb{R}^{n N}$ containing $(0,0)$. Let $\gamma$ be a Lorentz matrix in $Y, \gamma(0,0)=\eta$, and let $\beta$ be of degree $\varepsilon$. Let also $(\phi, \psi) \in H_{s, \delta+\frac{1}{2}}(\Sigma) \times H_{s-1, \delta+\frac{3}{2}}(\Sigma)$, where:

$$
s>\frac{n}{2}+2, \quad \delta>\varepsilon-\frac{n}{2}
$$

and let

$$
\{(\phi(x), \psi(x)) \mid x \in \Sigma\} \subset Y
$$

and $\Sigma$ be spacelike for $\gamma(\phi, \psi)$. Then:

(1) There exists $a \quad \theta>0$ and a unique solution $u \in H_{s, \delta}\left(\Omega_{\theta}\right)$ to:

$$
\gamma(u, D u) \cdot D^{2} u=\beta(u, D u),
$$

taking on $\Sigma$ the Cauchy data $(\phi, \psi)$ :

$$
u_{\mid \Sigma}=\phi, \quad D_{0} u_{\mid \Sigma}=\psi .
$$

Furthermore, $\gamma(u, D u)$ is a regularly hyperbolic metric on $\Omega_{\theta}$ and hence $\left(\Omega_{\theta}, \gamma(u, D u)\right)$ is a globally hyperbolic spacetime satisfying the largeness requirement of the boost problem.

(2) For every $\theta<1$ there exists a $d_{0}>0$ such that if $d<d_{0}$, where:

$$
d \equiv\|\phi\|_{H_{s, \delta+\frac{1}{2}(\Sigma)}}+\|\psi\|_{H_{s-1, \delta+\frac{s}{2}(\Sigma)}},
$$

then the above conclusion holds.

According to the above theorem the degree $\varepsilon$ of the function $\beta$ defines the minimal falloff of the Cauchy data at infinity required for the boost problem to have a solution. The quasilinear system behaves like a linear system at spacelike infinity when and only when the degree of $\beta$ is the least possible, namely $\varepsilon=0$. 
To apply Theorem 5.1 to the reduced Einstein equations we set:

$$
\gamma=\eta+u \text {. }
$$

Then expression (4.5) shows that:

$$
\beta(u, v)=P(\eta+u) \cdot v^{2} .
$$

We have $n=4$ and $N=10$ (dimension of the space of 2-contravariant symmetric tensors at a point in $\mathbb{R}^{4}$ ). The set $Y$ is given by:

$$
Y=\Lambda \times \mathbb{R}^{40},
$$

where $\Lambda$ is the set of 4-dimensional Lorentz matrices translated by $\eta$. $\Lambda$ is an open subset of $\mathbb{R}^{10}$ containing 0 . Both $\gamma$ and $\beta$ are $C^{\infty}$ (in fact analytic) functions of $(u, v)$ in $Y, \gamma$ is a Lorentz matrix in $Y$ and $\gamma(0,0)=\eta$. By Definition 5.1 and $5.2 \beta$ is of type $(0,2)$, degree 0 .

In Theorem 5.1 if $\gamma(u, v)$ is independent of $v$ and $Y$ is of the form $V \times \mathbb{R}^{n N}$ where $V$ is an open subset of $\mathbb{R}^{N}$, then it is clear from the proof of the theorem that the condition $s>n / 2+2$ can be weakened to $s>n / 2+1$. This in fact is the case for the reduced Einstein equations. Hence we obtain:

Lemma 5.1. Let $\phi(x)$ be a Lorentz matrix for each $x \in \Sigma$ and let $\Sigma$ be spacelike for $\phi$. Let also $(\phi-\eta, \psi) \in H_{s, \delta+\frac{1}{2}}(\Sigma) \times H_{s-1, \delta+\frac{3}{2}}(\Sigma)$, where $s \geqq 4, \delta>-2$. Then:

(1) There exists $a \theta>0$ and a unique solution $\gamma$ to the reduced Einstein equations in $\Omega_{\theta}, \gamma-\eta \in H_{s, \delta}\left(\Omega_{\theta}\right)$, taking on $\Sigma$ the Cauchy data $(\phi, \psi)$ :

$$
\gamma_{\mid \Sigma}=\phi, \quad D_{0} \gamma_{\mid \Sigma}=\psi \text {. }
$$

(2) For every $\theta<1$ there exists a $d_{0}>0$ such that if $d<d_{0}$, where:

$$
d \equiv\|\phi-\eta\|_{H_{s, \delta+\frac{1}{2}(\Sigma)}}+\|\psi\|_{H_{s-1, \delta+\frac{3}{2}(\Sigma)}},
$$

then the above conclusion holds.

The behaviour of the reduced Einstein equations at spacetime infinity is the same as that of a linear system due to the fact that the degree of $\beta$ is 0 . This is not the case for other non-linear hyperbolic systems of interest in physics. For example, the equation

$$
\square \phi=\lambda \phi^{3}
$$

has $\beta$ of type $(3,0)$, degree 1 . In the case of the reduced Yang-Mills equations, namely the Yang-Mills equations in a Lorentz gauge, $\beta(u, v)$ is of the form $u v+u^{3}$ which by Definition 5.3 is also of degree 1 . The behaviour of these equations at spacelike infinity is radically different from that of a linear system. In fact Theorem 5.1 does not allow one to conclude that there are solutions to the boost problem for the reduced Yang-Mills equations if the Cauchy data have non-vanishing Yang-Mills charge.

\section{The Boost Problem for the Einstein Equations}

In this paragraph we collect the results of paragraphs 4 and 5 into the following theorems (see introduction): 
Theorem 6.1 (Existence). Let $g$ be a riemannian metric and $k$ a 2-covariant symmetric tensorfield on $\Sigma$. If the pair $(g, k)$ satisfies the initial value constraints and if

$$
g-e \in H_{s, \delta+\frac{1}{2}}(\Sigma), \quad k \in H_{s-1, \delta+\frac{3}{2}}(\Sigma),
$$

where $s \geqq 4, \delta>-2$, then:

(1) There exists $a \theta>0$ and a solution $\gamma$ to the Einstein equations in $\Omega_{\theta}$, $\gamma-\eta \in H_{s, \delta}\left(\Omega_{\theta}\right)$, such that $(g, k)$ are respectively the first and second fundamental forms of $\Sigma$ relative to $\gamma$. In addition, $\gamma$ is a regularly hyperbolic metric on $\Omega_{\theta}$ and therefore $\left(\Omega_{\theta}, \gamma\right)$ is a globally hyperbolic spacetime satisfying the largeness requirement of the boost problem.

(2) For every $\theta<1$ there exists a $d_{0}>0$ such that if $d<d_{0}$, where

$$
d \equiv\|g-e\|_{H_{s, \delta+\frac{1}{2}(\Sigma)}}+\|k\|_{H_{s-1, \delta+\frac{3}{2}(\Sigma)}},
$$

the above conclusion holds.

Proof. Under the hypotheses of the theorem the Cauchy data $(\phi, \psi)$ for the reduced Einstein equations constructed from the initial data $(g, k)$ via (4.23), (4.24) satisfies the hypotheses of Lemma 5.1 as well as $\Gamma_{\mid \Sigma}^{\mu}=0$. Thus we have a unique regularly hyperbolic solution $\gamma$ to the reduced Einstein equations in $\Omega_{\theta}$ such that $\gamma-\eta \in H_{s, \delta}\left(\Omega_{\theta}\right)$. By Lemma 4.1 this solution also satisfies the full Einstein equations.

Theorem 6.2 (Uniqueness). Let $\gamma_{1}, \gamma_{2}$ be two regularly hyperbolic solutions to the Einstein equations in $\Omega_{\theta}$ such that the first and second fundamental forms of $\Sigma$ relative to $\gamma_{1}$ and $\gamma_{2}$ coincide. Let also $\gamma_{1}-\eta, \gamma_{2}-\eta \in H_{s+1, \delta}\left(\Omega_{\theta}\right)$ where $s \geqq 4, \delta>-2$. Then there exists an open subset $\Omega^{\prime} \subset \Omega_{\theta}, \Omega^{\prime} \supset \Sigma$, and a diffeomorphism $f: \Omega^{\prime} \rightarrow \Omega^{\prime \prime} \subset \Omega_{\theta}$, $f_{\mid \Sigma}=\mathrm{id}, f-\mathrm{id} \in H_{s+1, \delta-1}\left(\Omega^{\prime}\right)$, such that : $\gamma_{2}=f_{*} \gamma_{1}$ on $\Omega^{\prime \prime}$. Furthermore, $\left(\Omega^{\prime}, \gamma_{1}\right)$ (and therefore also $\left(\Omega^{\prime \prime}, \gamma_{2}\right)$ ) is a globally hyperbolic spacetime satisfying the largeness requirement of the boost problem.

Proof. Under the hypotheses of the theorem there exist, according to Theorem 4.3, diffeomorphisms $\xi_{1}: \Omega_{\theta_{1}} \rightarrow \Omega_{1}^{\prime}, \xi_{2}: \Omega_{\theta_{2}} \rightarrow \Omega_{2}^{\prime}, \xi_{1 \mid \Sigma}=\xi_{2 \mid \Sigma}=\mathrm{id}, \xi_{1}-\mathrm{id} \in H_{s+1, \delta-1}\left(\Omega_{\theta_{1}}\right)$, $\xi_{2}-\mathrm{id} \in H_{s+1, \delta-1}\left(\Omega_{\theta_{2}}\right)$, such that $\gamma_{1}^{\prime} \equiv \xi_{1 *} \gamma_{1}, \gamma_{2}^{\prime} \equiv \xi_{2 *} \gamma_{2}$ satisfy the harmonic condition on $\Omega_{1}^{\prime}, \Omega_{2}^{\prime}$ respectively and are both Gaussian on $\Sigma$. We can find some $\Omega_{\theta^{\prime}} \subset \Omega_{1}^{\prime} \cap \Omega_{2}^{\prime}$. Then $\gamma_{1}^{\prime}-\eta, \gamma_{2}^{\prime}-\eta \in H_{s, \delta}\left(\Omega_{\theta^{\prime}}\right)$ and $\gamma_{1}^{\prime}, \gamma_{2}^{\prime}$ are both solutions to the reduced Einstein equations in $\Omega_{\theta^{\prime}}$ having the same Cauchy data. It follows from Lemma 5.1 that $\gamma_{1}^{\prime}=\gamma_{2}^{\prime}$ on $\Omega_{\theta^{\prime}}$. Consequently, if we set $\Omega^{\prime}=\xi_{1}^{-1}\left(\Omega_{\theta^{\prime}}\right), \Omega^{\prime \prime}=\xi_{2}^{-1}\left(\Omega_{\theta^{\prime}}\right)$ and $f=\xi_{2}^{-1} \circ \xi_{1}$ the conclusion follows.

Theorem 6.2 says that two asymptotically flat developments of the same initial data which both satisfy the largeness requirement of the boost problem are extensions of a common asymptotically flat development of this data which also satisfies the largeness requirement of the boost problem. In view of the fact that global uniqueness is already known [6] for the Einstein equations, this theorem contains nothing new. It is included here for completeness of presentation. Theorem 6.1 is much more interesting; it shows that the maximal development of asymptotically flat initial data on $\Sigma$ includes complete spacelike hypersurfaces boosted relative to $\Sigma$. 
Finally, we wish to remark that the result of the boost problem can be strengthened somewhat by using the following argument. Given any initial data $(g, k)$ as in Theorem 6.1 , and given any $d_{0}>0$, we can find an $R$ such that the size $d\left(\Sigma-\bar{B}_{R}\right)$ of the restriction of $(g, k)$ to the complement of the closed ball of radius $R$,

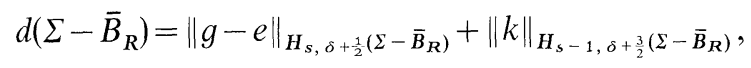

is less than $d_{0}$. Applying conclusion (2) of Theorem 6.1 one can then show that any asymptotically flat initial data set $(\Sigma, g, k)$ has a development $(\Omega, \gamma)$ satisfying the following:

For every $\lambda$ there exists an $R$ such that $\forall x \in \Sigma-\bar{B}_{R}$ :

$$
\chi^{ \pm}(x)>\lambda \tilde{d}(x) .
$$

Thus $\lambda \rightarrow \infty$ for $R \rightarrow \infty$ and the structure at spacelike infinity is complete. The same argument applies to Theorem 5.1.

\section{References}

1. Lichnerowicz, A.: Problèmes globaux en mécanique relativiste. Paris: Hermann 1939; Lichnerowicz, A. : J. Math. Pur. Appl. 23, 37-63 (1944)

2. Choquet-Bruhat, Y.: Acta Math. 88, 144-255 (1952) See also: Choquet-Bruhat, Y.: "Cauchy Problem". In: Gravitation; An introduction to current research. Witten, L. (ed.). New York: John Wiley 1962

3. Choquet-Bruhat, Y.: Ann. Inst. Henri Poincaré 8, 327-338 (1968) Choquet-Bruhat, Y.: C.R. Acad. Sci. Paris 272, 386-388 (1971) Choquet-Bruhat, Y.: G.R.G. 1, 359-362 (1971)

4. Lcray, J.: Hyperbolic differential equations. Institute for Advanced Study, Notes (1953)

5. Dionne, P.: J. Anal. Math. Jerusalem 10, 1-90 (1962)

6. Choquet-Bruhat, Y., Geroch, R.: Commun. Math. Phys. 14, 329-335 (1969)

7. Fischer, A., Marsden, J.: Commun. Math. Phys. 28, 1-38 (1972)

8. Hughes, T., Kato, T., Marsden, J.: Arch. Ration. Mech. Anal. 63, 273-294 (1977)

9. York, J.: Phys. Rev. Lett. 26, 1656-1658 (1971) York, J.: Phys. Rev. Lett. 28, 1082-1085 (1972)

York, J. : J. Math. Phys. 14, 456-464 (1973)

10. O’Murchadha, N., York, J.: Phys. Rev. D 10, 428-436 (1974)

11. Choquet-Bruhat, Y.: G.R.G. 5, 49-60 (1974)

12. Cantor, M.: Commun. Math. Phys. 57, 83-96 (1977)

13. Cantor, M.: J. Math. Phys. 20, 1741-1744 (1979)

14. Chaljub-Simon, A., Choquet-Bruhat, Y.: C.R. Acad. Sci. Paris 286, 917-920 (1978) Chaljub-Simon, A., Choquet-Bruhat, Y.: Global solutions of the Lichnerowicz equation in general relativity on an asymptotically euclidean complete manifold. Preprint (1979)

15. Hawking, S., Ellis, G.: The large scale structure of spacetime. Cambridge: Cambridge University Press 1973

16. Sommers, P.: J. Math. Phys. 19, 542-554 (1978)

17. Ashtekhar, A., Hansen, R.: J. Math. Phys. 19, 1542-1566 (1978)

18. Persides, S. : J. Math. Phys. 21, 135-151 (1980)

19. O'Murchadha, N.: Preprint (1978)

20. Adams, R.: Sobolev spaces. New York: Academic Press 1975

21. Choquet-Bruhat, Y., Christodoulou, D.: Elliptic systems in Hilbert spaces on manifolds which are euclidean at infinity. Acta Math. (to appear)

Choquet-Bruhat, Y., Christodoulou, D. : C.R. Acad. Sci. Paris 290, 781-785 (1980) 
22. Christodoulou, D.: The boost problem for weakly coupled quasilinear hyperbolic systems of the second order. J. Math. Pur. Appl. (to appear)

Christodoulou, D.: C.R. Acad. Sci. Paris 290, 641-644 (1980)

23. Cantor, M.: Compositio Math. 38, Fasc. 1, 3-35 (1979)

24. McOwen, R.: Commun. Pure Appl. Math. 32, 783-795 (1979)

25. Choquet, G., Choquet-Bruhat, Y.: C.R. Acad. Sci. Paris 287, 1047-1049 (1978)

26. Gilbarg, D., Trudinger, N.S.: "Elliptic partial differential equations of second order", Heidelberg: Springer 1977

27. Choquet-Bruhat, Y., DeWitt-Morette, C., Dillard-Bleick, M.: Analysis manifolds and physics. Amsterdam: North Holland 1977

28. Arnowitt, R., Deser, S., Misner, C.: In: Gravitation; An introduction to current research. Witten, L. (ed.). New York: John Wiley 1962

Communicated by A. Jaffe

Received September 17, 1980; in revised form March 16, 1981 